\title{
Helical buckling of a whirling conducting rod in a uniform magnetic field
}

\author{
J. Valverde \\ Department of Civil and Environmental Engineering, \\ University of California, Berkeley, USA \\ G.H.M. van der Heijden* \\ Centre for Nonlinear Dynamics, University College London, \\ Gower Street, London WC1E 6BT, UK
}

(Dated: June 24, 2018)

\begin{abstract}
We study the effect of a magnetic field on the behaviour of a conducting elastic rod subject to a novel set of boundary conditions that, in the case of a transversely isotropic rod, give rise to exact helical post-buckling solutions. The equations used are the geometrically exact Kirchhoff equations and both static (buckling) and dynamic (whirling) instability are considered. Critical loads are obtained explicitly and are given by a surprisingly simple formula. By solving the linearised equations about the (quasi-)stationary solutions we also find secondary instabilities described by (Hamiltonian-)Hopf bifurcations, the usual signature of incipient 'breathing' modes. The boundary conditions can also be used to generate and study helical solutions through traditional non-magnetic buckling due to compression, twist or whirl.
\end{abstract}

Keywords: rod mechanics, Kirchhoff equations, magnetic buckling, Hamiltonian-Hopf bifurcation, helical solutions

*Electronic address: g.heijden@ucl.ac.uk 


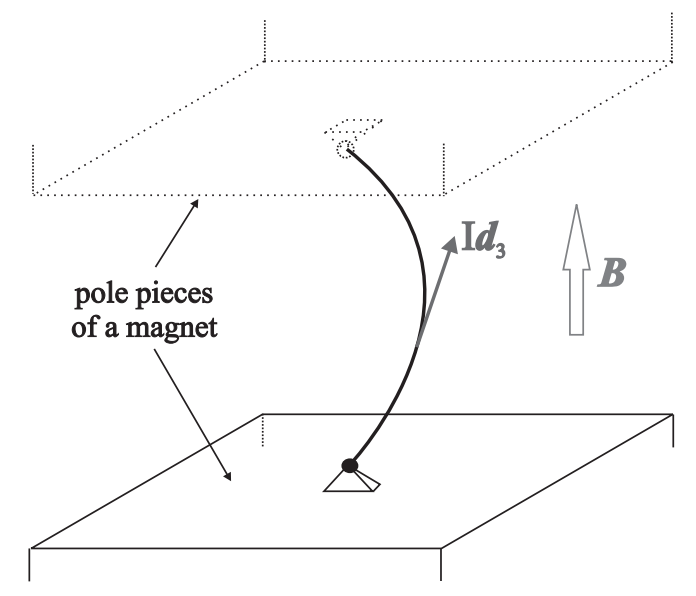

FIG. 1: Experimental setup for a conducting wire.

\section{INTRODUCTION}

A straight current-carrying wire held in tension between pole faces of a magnet is well known to buckle into a (roughly) helical configuration at a critical current (see Fig. 1). A photograph of this phenomenon is shown in Section 10.4.3 of [1], where a linear stability analysis is carried out for a simple string model. (A string is here meant to be a perfectly flexible elastic wire.) The problem was studied by Wolfe [2] by means of a rigorous bifurcation analysis for a (nonlinearly elastic) string suspended between fixed supports and placed in a uniform magnetic field directed parallel to the undeformed wire. He found that an infinite number of solution branches bifurcate from the trivial straight solution, much like in the Euler elastica under compressive load. In this case the non-trivial solutions are exact helices. That this should be so, is easily explained by the fact that the (Lorentz) body force is everywhere normal to the deformed configuration and hence the wire necessarily in a uniform state of tension. Some (statics) stability results (i.e., minimisation of the potential energy) were also obtained, indicating that the first branch of solutions is stable while the others are unstable.

In a subsequent paper Wolfe [3] extends the analysis to a uniformly rotating (whirling) string and shows again the existence of bifurcating branches of whirling non-trivial solutions. Due to centrifugal effects no closed-form solutions could be obtained in this case. This result

was further extended by Healey [4] using equivariant bifurcation theory in order to deal with the symmetries of the problem. 
Wolfe also considered a conducting rod in a uniform magnetic field [5]. In addition to extension a rod can undergo flexure, torsion and shear, and for the case of welded boundary conditions it was found that in certain cases bifurcation occurs, with the usual infinity of non-trivial equilibrium states. All the works cited above were content with showing the existence of bifurcating solutions and did not study their post-buckling behaviour.

In this paper we consider the post-buckling behaviour of a conducting rod. Wolfe considered welded boundary conditions in [5] and encountered degeneracies (even-dimensional eigenspaces) because of rotational symmetry of the problem. In previous work [6] we showed that further complications occur and that magnetic buckling of a welded transversely isotropic rod (i.e., a rod with unequal bending stiffnesses about the two principal axes of its cross-section) is described by a remarkably degenerate pitchfork bifurcation. Wolfe also reported numerical evidence of helical post-buckling solutions. However, exact helical solutions cannot be supported by (coaxial) welded boundary conditions. Here we formulate a novel set of what we call 'coat hanger' boundary conditions that do support (i.e., are compatible with) exact helical solutions, and show that subject to these boundary conditions an isotropic rod does indeed buckle (exclusively) into a helix, or more precisely, that there is an infinite series of helical modes bifurcating at increasing load, each successive mode having one more (half) helical turn. All helical solutions can be obtained explicitly and it is found that the pitchfork bifurcations for these coat hanger boundary conditions are non-degenerate and that the critical loads are given by a remarkably simple formula. Unlike in string buckling a rod does not require a tensile force in the trivial state, but we allow for such an applied force as well. The pertinent dimensionless parameter that governs buckling measures the product of current and magnetic field against the bending force.

We also study steady whirling solutions for which we introduce a rotating coordinate system. This extends Wolfe's analysis of whirling strings to whirling rods. An interesting feature of helical solutions is that since all points on a helix have equal distance to the whirling axis, and are therefore equally affected by centrifugal forces, solutions remain helical when spun. We perform a stability analysis by computing eigenvalues of the linearised boudary-value problem about a (quasi-stationary) whirling solution. For this we use a continuation (or homotopy) approach that takes advantage of the fact that exact expressions for the (imaginary) eigenvalues can be obtained in an appropriate limit (no spin, no magnetic field). The eigenvalues in this limit are then traced as system parameters are varied. 
Whirl tends to destabilise the helical solutions, but stable solutions can be obtained by adding the effect of internal viscoelastic damping. We find Hopf bifurcations on the first bifurcating branch where a stable whirling solution becomes unstable under an increase of the angular velocity. We also briefly consider anisotropic rods. Critical loads can still be obtained analytically, but these rods buckle into coiled but non-helical solutions. Secondary instabilities are found due to Hamiltonian-Hopf bifurcations, a common signature of 'breathing' or 'flutter' instabilities in mechanical systems.

Helical solutions are widely studied in a whole range of applications. Often these solutions are thought to arise through buckling of a straight rod under the action of end loads. However, as commented above, exact helical solutions are not supported by the usual set of boundary conditions. Consequently, boundary conditions are often not mentioned, or the rod is implicitly assumed to be infinitely long in order to prevent end effects [7]. One of the contributions of this paper is to present and highlight boundary conditions for an elastic rod that do support helical solutions. One could apply these experimentally if one was interested in generating or studying helical solutions in a finite-length rod.

The paper is organised as follows. In Section 2 we present the rod mechanics formulation, in which the magnetic field enters the force balance equation through the Lorentz body force. The coat hanger boundary conditions are introduced together with a sketch of a device that can be constructed to realise these boundary conditions in a testing rig. For the study of whirling solutions the equilibrium equations are transformed to a coordinate system rotating at constant angular velocity. We use numerical bifurcation and continuation methods to find the buckling loads and to compute post-buckling solution paths, both for the statics and dynamics case. After the nondimensionalisation in Section 3, the linearisation is presented in Section 4. Section 5 first presents analytical stability results for the statics case and then introduces our continuation approach to numerical stability analysis of the full system. In Section 6 results are presented in the form of bifurcation diagrams and curves of Hopf bifurcations in appropriate parameter planes. Conclusions are drawn in Section 7 and the study closes with two Appendices: one giving details about the linearised system of equations and one deriving exact buckling results for helical solutions. The latter is complementary to the bifurcation analysis (not assuming any shape) in Section 5; together these analyses give a complete picture of helical magnetic buckling. 


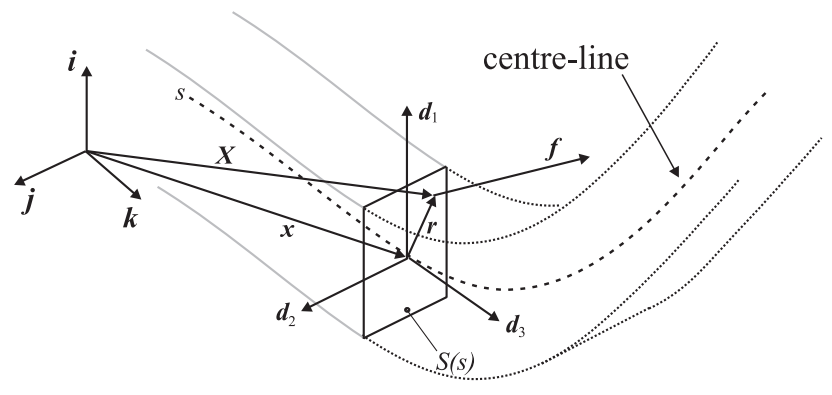

FIG. 2: Cosserat model of a rod.

\section{THE ROD MECHANICS MODEL}

We describe the elastic behaviour of a conducting cable by the Kirchhoff equations for the dynamics of thin rods. The rod is assumed to be uniform, inextensible, unshearable and intrinsically straight and prismatic. The assumptions of inextensibility and unshearability are appropriate for thin rods with relatively low external (here electrodynamic) forces. For the background of the Kirchhoff equations the reader is referred to [8, 9]. These equations were also used in [6] and [10] to analyse the dynamics of a spinning tether.

Let $\boldsymbol{x}$ denote the position of the rod's centreline and let $\left\{\boldsymbol{d}_{1}, \boldsymbol{d}_{2}, \boldsymbol{d}_{3}\right\}$ be a right-handed orthonormal frame of directors (the Cosserat triad) defined at each point along the centreline. Since the centreline is assumed to be inextensible we can take $\boldsymbol{d}_{3}$ in the direction of the local tangent:

$$
\boldsymbol{x}^{\prime}(s, t)=\boldsymbol{d}_{3}(s, t)
$$

where the prime denotes differentiation with respect to arclength $s$ measured along the centreline, and $t$ is time. The directors $\boldsymbol{d}_{1}$ and $\boldsymbol{d}_{2}$ will be taken to point along the principal bending axes of the cross-section (see Fig. 2). The unstressed rod is taken to lie along the basis vector $\boldsymbol{k}$ of a fixed inertial frame $\{\boldsymbol{i}, \boldsymbol{j}, \boldsymbol{k}\}$.

Looking at Fig. 2 we note that the position vector of an arbitrary point of the rod can be expressed as

$$
\begin{aligned}
\boldsymbol{X}\left(s, \xi_{1}, \xi_{2}, t\right) & =\boldsymbol{x}(s, t)+\xi_{1} \boldsymbol{d}_{1}(s, t)+\xi_{2} \boldsymbol{d}_{2}(s, t) \\
& =\boldsymbol{x}(s, t)+\boldsymbol{r}\left(s, \xi_{1}, \xi_{2}, t\right),
\end{aligned}
$$

where $\left(\xi_{1}, \xi_{2}\right)$ are the components of $\boldsymbol{r}$ in the cross-section relative to $\left\{\boldsymbol{d}_{1}(s), \boldsymbol{d}_{2}(s)\right\}$. The rod is thus viewed as a set of infinitesimal slices centred at all $s$. A one-dimensional description 
will be obtained by averaging of forces and moments over each cross-section. The internal traction, which is the projection of the stress tensor onto the cross-sectional plane, is given by a force which we denote by $\boldsymbol{f}=\boldsymbol{f}\left(s, \xi_{1}, \xi_{2}, t\right)$ (see Fig. 2). The resultant elastic force exerted in a section $S(s)$ is given by

$$
\boldsymbol{F}(s, t)=\int_{S(s)} \boldsymbol{f}\left(s, \xi_{1}, \xi_{2}, t\right) \mathrm{d} S
$$

where $\mathrm{d} S$ is an infinitesimal area element. This force can be expressed in the director basis as $\boldsymbol{F}=\sum_{i=1}^{3} F_{i} \boldsymbol{d}_{i}$. The resultant moment in the section $S(s)$ is given by

$$
\boldsymbol{M}(s, t)=\int_{S(s)} \boldsymbol{r}\left(s, \xi_{1}, \xi_{2}, t\right) \times \boldsymbol{f}\left(s, \xi_{1}, \xi_{2}, t\right) \mathrm{d} S,
$$

and will be expressed as $\boldsymbol{M}=\sum_{i=1}^{3} M_{i} \boldsymbol{d}_{i}$.

The rod is assumed to carry an electric current for which we can write

$$
\boldsymbol{I}=I \boldsymbol{x}^{\prime}=I \boldsymbol{d}_{3}
$$

Here we have assumed the current to have the same direction as the rod, which is consistent with a one-dimensional rod theory. It amounts to the assumption that the cross-section of the conducting wire is small enough to make currents within the cross-section (eddy currents) induced by the motion negligible. The current $\boldsymbol{I}$ interacts with the magnetic field $\boldsymbol{B}_{0}$ to generate a (Lorentz) body force given by

$$
\boldsymbol{F}_{L}=I \boldsymbol{d}_{3} \times \boldsymbol{B}_{0}
$$

Following [2] we assume the magnetic field to be uniform and directed along the unstressed rod, i.e.,

$$
\boldsymbol{B}_{0}=B_{0} \boldsymbol{k}
$$

The balancing of forces and moments across an infinitesimal rod element then yields the following set of partial differential equations [8, 9]:

$$
\begin{gathered}
\boldsymbol{F}^{\prime}+I B_{0} \boldsymbol{d}_{3} \times \boldsymbol{k}=\rho A \ddot{\boldsymbol{x}} \\
\boldsymbol{M}^{\prime}+\boldsymbol{d}_{3} \times \boldsymbol{F}=\rho\left(I_{2} \boldsymbol{d}_{1} \times \ddot{\boldsymbol{d}}_{1}+I_{1} \boldsymbol{d}_{2} \times \ddot{\boldsymbol{d}}_{2}\right)
\end{gathered}
$$

where $\rho$ is the (volumetric) mass density, $A$ the cross-sectional area, $I_{1}$ and $I_{2}$ the second moment of area of the cross-section about $\boldsymbol{d}_{1}$ and $\boldsymbol{d}_{2}$ respectively, and ( ) denotes differentiation with respect to time. 
For a closed system of equations these balance equations need to be supplemented by constitutive relations that characterise the material behaviour of the rod. We assume the rod to be made of homogeneous isotropic linear viscoelastic material so that stress-strain relations, based on a model by Valverde et al. [10], are

$$
\begin{aligned}
& M_{1}=E I_{1}\left(\kappa_{1}+\gamma_{v} \dot{\kappa}_{1}\right), \\
& M_{2}=E I_{2}\left(\kappa_{2}+\gamma_{v} \dot{\kappa}_{2}\right), \\
& M_{3}=G J\left(\kappa_{3}+\gamma_{v} \dot{\kappa}_{3}\right),
\end{aligned}
$$

where $\kappa_{1}$ and $\kappa_{2}$ are the curvatures about $\boldsymbol{d}_{1}$ and $\boldsymbol{d}_{2}$, respectively, while $\kappa_{3}$ is the twist about $\boldsymbol{d}_{3}$. The constant $\gamma_{v}$ is the viscoelastic coefficient of the material, $E$ is Young's modulus, $G$ is the shear modulus and $J$ is the second moment of area of the section about $\boldsymbol{d}_{3}$. We shall assume that the section is symmetric with respect to the principal axes, in which case $J=I_{1}+I_{2}$.

The $\kappa_{i}$ are the components of the curvature vector

$$
\boldsymbol{\kappa}=\sum_{i=1}^{3} \kappa_{i} \boldsymbol{d}_{i},
$$

which governs the evolution in space of the frame of directors as one moves along the centreline:

$$
\boldsymbol{d}_{i}^{\prime}=\boldsymbol{\kappa} \times \boldsymbol{d}_{i} \quad(i=1,2,3) .
$$

The constitutive relations (10) can be used to replace the $\kappa_{i}$ in (12) by moments, after which the equations (1), (8), (9) and (12) form a system of 18 differential equations for the 18 unknowns $\left(\boldsymbol{x}, \boldsymbol{F}, \boldsymbol{M}, \boldsymbol{d}_{1}, \boldsymbol{d}_{2}, \boldsymbol{d}_{3}\right)$.

Remark: We ignore in this study secondary electrodynamic effects (such as an induced emf and hence additional current in the conductor) as a result of the motion of the wire in the magnetic field [11]. Since we are considering a steadily rotating wire these effects would be null on the configuration of the wire. However, the same would not be true for the stability analysis, which considers arbitrary time-dependent perturbations. We assume that these induction effects are negligible. 


\section{A. Equations of motion in a uniformly rotating frame}

We shall also be interested in steadily rotating solutions and therefore we transform the equilibrium equations (8) and (9) to a coordinate frame $\left\{\boldsymbol{e}_{1}, \boldsymbol{e}_{2}, \boldsymbol{e}_{3}\right\}$ that rotates with constant angular velocity $\boldsymbol{\omega}=\omega \boldsymbol{k}$ about the $\boldsymbol{k}$ axis (and the axis of the rod in its trivial unstressed state). Noting that the derivative with respect to time of an arbitrary vector $\boldsymbol{V}(s, t)$ is given by

$$
\left.\frac{d \boldsymbol{V}(s, t)}{d t}\right|_{i}=\left.\frac{d \boldsymbol{V}(s, t)}{d t}\right|_{m}+\boldsymbol{\omega} \times \boldsymbol{V}(s, t),
$$

where $\left.\frac{d}{d t}\right|_{i}$ indicates the derivative with respect to time in the inertial frame and $\left.\frac{d}{d t}\right|_{m}$ stands for the derivative with respect to time in the moving frame, the equations (8) and (9) expressed relative to $\left\{\boldsymbol{e}_{1}, \boldsymbol{e}_{2}, \boldsymbol{e}_{3}\right\}$ become

$$
\begin{gathered}
\boldsymbol{F}^{\prime}+I B_{0} \boldsymbol{d}_{3} \times \boldsymbol{e}_{3}=\rho A(\ddot{\boldsymbol{x}}+2 \boldsymbol{\omega} \times \dot{\boldsymbol{x}}+\boldsymbol{\omega} \times(\boldsymbol{\omega} \times \boldsymbol{x})) \\
\boldsymbol{M}^{\prime}+\boldsymbol{d}_{3} \times \boldsymbol{F}=\rho I_{2}\left(\boldsymbol{d}_{1} \times \ddot{\boldsymbol{d}}_{1}+2 \boldsymbol{d}_{1} \times\left(\boldsymbol{\omega} \times \dot{\boldsymbol{d}}_{1}\right)+\left(\boldsymbol{\omega} \cdot \boldsymbol{d}_{1}\right)\left(\boldsymbol{d}_{1} \times \boldsymbol{\omega}\right)\right) \\
+\rho I_{1}\left(\boldsymbol{d}_{2} \times \ddot{\boldsymbol{d}}_{2}+2 \boldsymbol{d}_{2} \times\left(\boldsymbol{\omega} \times \dot{\boldsymbol{d}}_{2}\right)+\left(\boldsymbol{\omega} \cdot \boldsymbol{d}_{2}\right)\left(\boldsymbol{d}_{2} \times \boldsymbol{\omega}\right)\right) .
\end{gathered}
$$

The second term on the right-hand side of equation (14) is the Coriolis force, while the third term is the centrifugal force, as a result of the rotating coordinate system.

Steadily rotating (whirling) solutions satisfy the equations (14) and (15) with the dotted variables set to zero:

$$
\begin{gathered}
\boldsymbol{F}^{\prime}+I B_{0} \boldsymbol{d}_{3} \times \boldsymbol{e}_{3}=\rho A \boldsymbol{\omega} \times(\boldsymbol{\omega} \times \boldsymbol{x}), \\
\boldsymbol{M}^{\prime}+\boldsymbol{d}_{3} \times \boldsymbol{F}=\rho I_{2}\left(\boldsymbol{\omega} \cdot \boldsymbol{d}_{1}\right)\left(\boldsymbol{d}_{1} \times \boldsymbol{\omega}\right)+\rho I_{1}\left(\boldsymbol{\omega} \cdot \boldsymbol{d}_{2}\right)\left(\boldsymbol{d}_{2} \times \boldsymbol{\omega}\right) .
\end{gathered}
$$

The other equations (11) and (12) do not change their form, but all vectors are now to be considered as expressed relative to the rotating frame $\left\{\boldsymbol{e}_{1}, \boldsymbol{e}_{2}, \boldsymbol{e}_{3}\right\}$. Statical solutions are simply obtained by setting $\omega$ equal to zero.

For a well-posed problem the final 18 ODEs require 18 boundary conditions to be specified, which we do next.

\section{B. Coat hanger boundary conditions}

Helical solutions in rods are usually studied in infinitely long rods, which avoids the need for imposing boundary conditions. Indeed, it is not immediately clear how an exact helix 


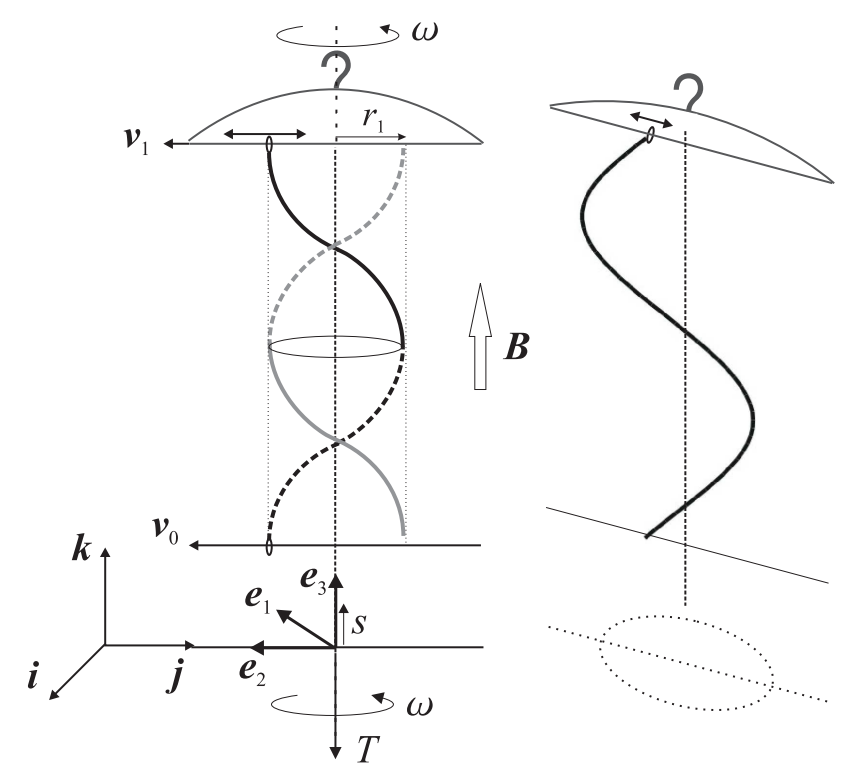

FIG. 3: Coat hanger boundary conditions.

can be supported: the boundary conditions cannot be simply welded as no two points on a helix have coaxial tangents, nor can they be simply pinned because a helix has curvature and therefore carries a bending moment. Here we formulate a set of boundary conditions that support exact helical solutions. We call them coat hanger boundary conditions, for obvious reasons.

Consider Fig. 3 where a rod is suspended between two axes $\boldsymbol{v}_{0}$ (at $s=0$ ) and $\boldsymbol{v}_{1}$ (at $s=1$ ) lying in two parallel planes normal to $\boldsymbol{e}_{3}$. Axis $\boldsymbol{v}_{1}$ is taken to be fixed in space, while $\boldsymbol{v}_{0}$ is free to move along $\boldsymbol{e}_{3}$. We assume the axes to have a fixed relative rotation $\chi$, i.e., $\boldsymbol{v}_{0} \cdot \boldsymbol{v}_{1}=\cos \chi$. The rod is free to hinge about and slide along both $\boldsymbol{v}_{0}$ and $\boldsymbol{v}_{1}$. For definiteness we assume that the rod is mounted in such a way that both axes $\boldsymbol{v}_{0}$ and $\boldsymbol{v}_{1}$ are directed along the vector $\boldsymbol{d}_{2}$ in the rod's cross-section. This situation is described by the 
following boundary conditions:

$$
\begin{aligned}
\boldsymbol{d}_{1}(0, t) \cdot \boldsymbol{v}_{0} & =0, \\
\boldsymbol{d}_{3}(0, t) \cdot \boldsymbol{v}_{0} & =0, \\
\boldsymbol{M}(0, t) \cdot \boldsymbol{v}_{0} & =0, \\
\boldsymbol{F}(0, t) \cdot \boldsymbol{v}_{0} & =0, \\
\boldsymbol{F}(0, t) \cdot \boldsymbol{e}_{3} & =T, \\
\boldsymbol{x}(0, t) \cdot\left(\boldsymbol{v}_{0} \times \boldsymbol{e}_{3}\right) & =0,
\end{aligned}
$$

at $s=0$, and

$$
\begin{aligned}
\boldsymbol{d}_{1}(L, t) \cdot \boldsymbol{v}_{1} & =0, \\
\boldsymbol{d}_{3}(L, t) \cdot \boldsymbol{v}_{1} & =0, \\
\boldsymbol{M}(L, t) \cdot \boldsymbol{v}_{1} & =0, \\
\boldsymbol{F}(L, t) \cdot \boldsymbol{v}_{1} & =0, \\
\boldsymbol{x}(L, t) \cdot\left(\boldsymbol{v}_{1} \times \boldsymbol{e}_{3}\right) & =0, \\
z(L, t) & =L,
\end{aligned}
$$

at $s=1$, where the position vector has been decomposed as $\boldsymbol{x}=x \boldsymbol{e}_{1}+y \boldsymbol{e}_{2}+z \boldsymbol{e}_{3}$ and $T$ is an applied end force (positive for tension). Conditions (23) and (28) restrict the movement of the ends of the rod to the planes spanned by $\left(\boldsymbol{v}_{0}, \boldsymbol{e}_{3}\right)$ and $\left(\boldsymbol{v}_{1}, \boldsymbol{e}_{3}\right)$, respectively. To these 12 conditions we have to add conditions that ensure the orthonormality of the director basis, for which we can take

$$
\begin{aligned}
& \boldsymbol{d}_{1}(0, t) \cdot \boldsymbol{d}_{1}(0, t)=1, \\
& \boldsymbol{d}_{2}(0, t) \cdot \boldsymbol{d}_{2}(0, t)=1, \\
& \boldsymbol{d}_{3}(0, t) \cdot \boldsymbol{d}_{3}(0, t)=1, \\
& \boldsymbol{d}_{1}(0, t) \cdot \boldsymbol{d}_{2}(0, t)=0, \\
& \boldsymbol{d}_{1}(0, t) \cdot \boldsymbol{d}_{3}(0, t)=0 \\
& \boldsymbol{d}_{2}(0, t) \cdot \boldsymbol{d}_{3}(0, t)=0
\end{aligned}
$$

for a total of 18 boundary conditions, as required.

We shall take $\chi=0$ so that the initial rod, lying straight along $\boldsymbol{e}_{3}$, is untwisted. This choice implies that any helical solutions will have an integer number of half helical periods. 
Also, the directors, and hence the cross-section of the rod, will make a half-integer number of turns between $s=0$ and $s=L$. Without loss of generality we may choose $\boldsymbol{v}_{0}=\boldsymbol{v}_{1}=\boldsymbol{e}_{2}$, so that at the ends of the initial rod the directors $\left\{\boldsymbol{d}_{1}, \boldsymbol{d}_{2}, \boldsymbol{d}_{3}\right\}$ are aligned with $\left\{\boldsymbol{e}_{1}, \boldsymbol{e}_{2}, \boldsymbol{e}_{3}\right\}$.

Since $\boldsymbol{v}_{0}$ and $\boldsymbol{v}_{1}$ are chosen aligned, there is a rigid-body degree of freedom of translation of any solution along these axes. To eliminate this degeneracy we replace condition (27) above by

$$
\boldsymbol{x}(L, t) \cdot \boldsymbol{v}_{1}=r_{1},
$$

where the slide $r_{1}$ along $\boldsymbol{v}_{1}$ (see Fig. (3) is chosen as follows. First note that a helix is a curve of constant axial radius $r=\sqrt{x^{2}+y^{2}}$ and (total) curvature $\kappa=\sqrt{\kappa_{1}^{2}+\kappa_{2}^{2}}$. The two are related by $\kappa r=\sin ^{2} \theta$, where $\theta$ is the helical angle defined by $\boldsymbol{d}_{3} \cdot \boldsymbol{e}_{3}=\cos \theta$ if $\boldsymbol{e}_{3}$ is along the axis of the helix (the angle $\frac{\pi}{2}-\theta$ is usually called the pitch angle). Since the rod is hinged about $\boldsymbol{d}_{2}$, we have $\kappa_{2}(L, t)=0$. So, in order to ensure that any bifurcating helix is centred at $\boldsymbol{e}_{3}$ we take

$$
r_{1}=\frac{1-\left(\boldsymbol{d}_{3}(L, t) \cdot \boldsymbol{e}_{3}\right)^{2}}{\kappa_{1}(L, t)},
$$

giving a nonlinear boundary condition. Note that $\kappa_{1}$ can here be taken with its sign, so that (31) also specifies which way the rod moves along $\boldsymbol{v}_{1}$. When the rod buckles, the axis $\boldsymbol{v}_{0}$ lifts up and the rod is free to find its own radius $r=\left|r_{1}\right|$. We stress that condition (31) has no effect on the bifurcation behaviour. In particular, it does not suppress any non-helical solutions. It merely ensures that if a helical solution bifurcates it will be centred at the axis of rotation. This is important when we start rotating the axes $\boldsymbol{v}_{0}$ and $\boldsymbol{v}_{1}$ about $\boldsymbol{e}_{3}$. A centred helix will experience a uniform centrifugal force and is therefore expected to remain helical.

Of course the above coat hanger boundary conditions merely allow for helical solutions. They need not exist. However, if the equilibrium equations do have helical solutions and one-parameter curves of such solutions intersect the trivial path of straight solutions, then one might expect to detect them as (pitchfork) bifurcations at critical buckling loads. The results presented in Section VI show that this is indeed the case. 


\section{NONDIMENSIONALISATION}

We make the system of equations dimensionless by scaling the variables in the following way

$$
\begin{aligned}
& \omega_{c}=\sqrt{\frac{E I_{1}}{\rho A L^{4}}}, \quad \bar{t}=t \omega_{c}, \quad \bar{s}=\frac{s}{L} \in[0,1], \quad \bar{\omega}=\frac{\omega}{\omega_{c}}, \quad \overline{\boldsymbol{x}}=\frac{\boldsymbol{x}}{L}, \\
& \overline{\boldsymbol{F}}=\boldsymbol{F} \frac{L^{2}}{E I_{1}}, \quad \bar{T}=T \frac{L^{2}}{E I_{1}}, \quad \overline{\boldsymbol{M}}=\boldsymbol{M} \frac{L}{E I_{1}}, \quad \overline{\boldsymbol{\kappa}}=\boldsymbol{\kappa} L .
\end{aligned}
$$

Here $\omega_{c}$ is a reference characteristic bending frequency of the rod.

With this nondimensionalisation the equations become (dropping the overbars for simplicity and letting a prime denote $\frac{d}{d \bar{s}}$ and an overdot $\frac{d}{d \bar{t}}$ ):

$$
\begin{gathered}
\boldsymbol{F}^{\prime}+B \boldsymbol{d}_{3} \times \boldsymbol{k}=\ddot{\boldsymbol{x}}+2 \boldsymbol{\omega} \times \dot{\boldsymbol{x}}+\boldsymbol{\omega} \times(\boldsymbol{\omega} \times \boldsymbol{x}), \\
\boldsymbol{M}^{\prime}+\boldsymbol{d}_{3} \times \boldsymbol{F}=P\left[R\left(\boldsymbol{d}_{1} \times \ddot{\boldsymbol{d}}_{1}+2 \boldsymbol{d}_{1} \times \boldsymbol{\omega} \times \dot{\boldsymbol{d}}_{1}+\left(\boldsymbol{\omega} \cdot \boldsymbol{d}_{1}\right)\left(\boldsymbol{d}_{1} \times \boldsymbol{\omega}\right)\right)\right. \\
\left.+\left(\boldsymbol{d}_{2} \times \ddot{\boldsymbol{d}}_{2}+2 \boldsymbol{d}_{2} \times \boldsymbol{\omega} \times \dot{\boldsymbol{d}}_{2}+\left(\boldsymbol{\omega} \cdot \boldsymbol{d}_{2}\right)\left(\boldsymbol{d}_{2} \times \boldsymbol{\omega}\right)\right)\right] \\
\boldsymbol{x}^{\prime}=\boldsymbol{d}_{3} \\
\boldsymbol{d}_{i}^{\prime}=\boldsymbol{\kappa} \times \boldsymbol{d}_{i}
\end{gathered}
$$

and the constitutive relations can be written as

$$
\boldsymbol{M}=\left(\kappa_{1}+\gamma \dot{\kappa_{1}}\right) \boldsymbol{d}_{1}+R\left(\kappa_{2}+\gamma \dot{\kappa_{2}}\right) \boldsymbol{d}_{2}+\frac{\Gamma(1+R)}{2}\left(\kappa_{3}+\gamma \dot{\kappa_{3}}\right) \boldsymbol{d}_{3}
$$

where the dimensionless parameters are

$$
P=\frac{I_{1}}{A L^{2}}, \quad R=\frac{I_{2}}{I_{1}}, \quad B=\frac{B_{0} I L^{3}}{E I_{1}}, \quad \Gamma=\frac{2 G}{E}, \quad \gamma=\gamma_{v} \omega_{c}
$$

and $\left(\frac{1}{\Gamma}-1\right)$ is equal to Poisson's ratio. For the boundary conditions we can still use (18) to (29) if we assume that they now refer to dimensionless variables and that the right-hand conditions are imposed at $\bar{s}=1$.

\section{PERTURBATION SCHEME - LINEARISATION}

We consider whirling solutions (relative equilibria) that are stationary in the moving frame $\left\{\boldsymbol{e}_{1}, \boldsymbol{e}_{2}, \boldsymbol{e}_{3}\right\}$. Such solutions are found by solving the set of equations (34)-(38) with the dotted variables set to zero (thus obtaining an ODE). To study their stability we linearise 
the full PDE (34)-(38) about these whirling solutions. We follow the approach in [10], which is similar to approaches in [7, 12]. The stability of static (non-whirling) solutions can be investigated by simply setting the angular velocity $\omega$ to zero.

We start our perturbation analysis by writing

$$
\boldsymbol{d}_{i}(s, t)=\boldsymbol{d}_{i}^{0}(s)+\delta \boldsymbol{d}_{i}^{t}(s, t)+O\left(\delta^{2}\right), \quad i=1,2,3,
$$

where $\boldsymbol{d}_{i}^{0}(s)$ are the basis vectors of a quasi-stationary solution, $\boldsymbol{d}_{i}^{t}(s, t)$ are the basis vectors of a time-dependent perturbation and $\delta$ is a small bookkeeping parameter introduced to separate scales. Note that, in order to preserve orthonormality to $O(\delta)\left(\boldsymbol{d}_{i} \cdot \boldsymbol{d}_{j}=\delta_{i j}+O\left(\delta^{2}\right)\right)$, we must have

$$
\boldsymbol{d}_{i}^{t}(s, t)=\sum_{j=1}^{3} A_{i j}(s, t) \boldsymbol{d}_{j}^{0}(s), \quad i=1,2,3,
$$

where the matrix $A_{i j}$ is skew-symmetric and can be written as

$$
\boldsymbol{A}=\left(\begin{array}{ccc}
0 & \alpha_{3} & -\alpha_{2} \\
-\alpha_{3} & 0 & \alpha_{1} \\
\alpha_{2} & -\alpha_{1} & 0
\end{array}\right)
$$

Thus, the nine components of the director basis perturbation are described by only three independent parameters, and if we introduce

$$
\boldsymbol{\alpha}=\left(\alpha_{1}, \alpha_{2}, \alpha_{3}\right)^{T}
$$

(with respect to the unperturbed director basis) then the perturbed director basis can be expressed as

$$
\boldsymbol{d}_{i}(s, t)=\boldsymbol{d}_{i}^{0}(s)+\delta \boldsymbol{\alpha}(s, t) \times \boldsymbol{d}_{i}^{0}(s)+O\left(\delta^{2}\right), \quad i=1,2,3 .
$$

Using (44), the perturbation of an arbitrary vector $\boldsymbol{V}=\sum_{i=1}^{3} V_{i} \boldsymbol{d}_{i}$ can be written on the basis $\left\{\boldsymbol{d}_{1}^{0}, \boldsymbol{d}_{2}^{0}, \boldsymbol{d}_{3}^{0}\right\}$ as

$$
\boldsymbol{V}=\boldsymbol{V}^{0}+\delta \boldsymbol{V}^{t}+O\left(\delta^{2}\right)=\sum_{i=1}^{3}\left[V_{i}^{0}+\delta\left(V_{i}^{t}+\left(\boldsymbol{\alpha} \times \boldsymbol{V}^{0}\right)_{i}\right)\right] \boldsymbol{d}_{i}^{0}+O\left(\delta^{2}\right),
$$

where ()$_{i}$ denotes the component along $\boldsymbol{d}_{i}^{0}$ and time and space dependence of the variables have been suppressed for the sake of simplicity [10].

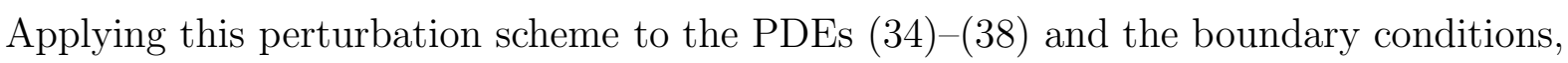
we arrive at an $O(1)$ nonlinear ODE for the quasi-stationary solutions and an $O(\delta)$ linear PDE governing their stability. 


\section{A. The $O(1)$ equations - quasi-stationary whirl}

The $O(1)$ equations are time-independent. Recalling that $\boldsymbol{\omega}=\boldsymbol{\omega}_{3}$, we find the $O(1)$ terms of the linear momentum equation (34), projected on the director basis $\left\{\boldsymbol{d}_{1}^{0}, \boldsymbol{d}_{2}^{0}, \boldsymbol{d}_{3}^{0}\right\}$, to give

$$
\begin{aligned}
\left(F_{1}^{0}\right)^{\prime}-F_{2}^{0} \kappa_{3}^{0}+F_{3}^{0} \kappa_{2}^{0}+B\left(d_{32}^{0} d_{11}^{0}-d_{31}^{0} d_{12}^{0}\right) & =-\omega^{2}\left(x^{0} d_{11}^{0}+y^{0} d_{12}^{0}\right), \\
\left(F_{2}^{0}\right)^{\prime}-F_{3}^{0} \kappa_{1}^{0}+F_{1}^{0} \kappa_{3}^{0}+B\left(d_{32}^{0} d_{21}^{0}-d_{31}^{0} d_{22}^{0}\right) & =-\omega^{2}\left(x^{0} d_{21}^{0}+y^{0} d_{22}^{0}\right), \\
\left(F_{3}^{0}\right)^{\prime}-F_{1}^{0} \kappa_{2}^{0}+F_{2}^{0} \kappa_{1}^{0} & =-\omega^{2}\left(x^{0} d_{31}^{0}+y^{0} d_{32}^{0}\right),
\end{aligned}
$$

where subscripts are used to indicate components relative to the basis vectors $\left\{\boldsymbol{d}_{1}^{0}, \boldsymbol{d}_{2}^{0}, \boldsymbol{d}_{3}^{0}\right\}$ (but the $\boldsymbol{d}_{i}^{0}$ components are relative to $\left\{\boldsymbol{e}_{1}, \boldsymbol{e}_{2}, \boldsymbol{e}_{3}\right\}$ ). Similarly, the $O(1)$ term of the angular momentum equation (35), projected on the director basis $\left\{\boldsymbol{d}_{1}^{0}, \boldsymbol{d}_{2}^{0}, \boldsymbol{d}_{3}^{0}\right\}$ gives

$$
\begin{aligned}
& \left(M_{1}^{0}\right)^{\prime}=\frac{2 M_{3}^{0} M_{2}^{0}}{\Gamma(1+R)}-\frac{M_{2}^{0} M_{3}^{0}}{R}+F_{2}^{0}+P \omega^{2} d_{23}^{0}\left(d_{22}^{0} d_{11}^{0}-d_{21}^{0} d_{12}^{0}\right), \\
& \left(M_{2}^{0}\right)^{\prime}=-\frac{2 M_{3}^{0} M_{1}^{0}}{\Gamma(1+R)}+M_{1}^{0} M_{3}^{0}-F_{1}^{0}+P R \omega^{2} d_{13}^{0}\left(d_{21}^{0} d_{12}^{0}-d_{11}^{0} d_{22}^{0}\right), \\
& \left(M_{3}^{0}\right)^{\prime}=\frac{M_{2}^{0} M_{1}^{0}}{R}-M_{1}^{0} M_{2}^{0}+P R \omega^{2} d_{13}^{0}\left(d_{12}^{0} d_{31}^{0}-d_{11}^{0} d_{32}^{0}\right)+P \omega^{2} d_{23}^{0}\left(d_{22}^{0} d_{31}^{0}-d_{21}^{0} d_{32}^{0}\right) .
\end{aligned}
$$

The $O(1)$ term of equation (36) can be expressed as

$$
\left(\boldsymbol{x}^{0}\right)^{\prime}=\boldsymbol{d}_{3}^{0},
$$

and the twist equation (37) by

$$
\left(\boldsymbol{d}_{i}^{0}\right)^{\prime}=\boldsymbol{\kappa}^{0} \times \boldsymbol{d}_{i}^{0}, \quad i=1,2,3,
$$

where $\boldsymbol{\kappa}^{0}=\sum_{j=1}^{3} \kappa_{j}^{0} \boldsymbol{d}_{j}^{0}$. The $O(1)$ term of constitutive relations (38) can be expressed as

$$
M_{1}^{0}=\kappa_{1}^{0}, \quad M_{2}^{0}=R \kappa_{2}^{0}, \quad M_{3}^{0}=\frac{\Gamma(1+R)}{2} \kappa_{3}^{0},
$$

which can be used to express the $\kappa_{i}^{0}$ in (53) in terms of the moments $M_{i}^{0}$. 
Proceeding in the same way, the $O(1)$ part of the boundary conditions is given by

$$
\begin{aligned}
& \boldsymbol{d}_{1}^{0}(0) \cdot \boldsymbol{v}_{0}=0, \\
& \boldsymbol{d}_{3}^{0}(0) \cdot \boldsymbol{v}_{0}=0, \\
& \boldsymbol{}^{0}(0) \cdot \boldsymbol{v}_{0}=0, \\
& \boldsymbol{F}^{0}(0) \cdot \boldsymbol{v}_{0}=0, \\
& \boldsymbol{F}^{0}(0) \cdot \boldsymbol{e}_{3}=T, \\
& \boldsymbol{x}^{0}(0) \cdot\left(\boldsymbol{v}_{0} \times \boldsymbol{e}_{3}\right)=0, \\
& \boldsymbol{d}_{1}^{0}(1) \cdot \boldsymbol{v}_{1}=0 \\
& \boldsymbol{d}_{3}^{0}(1) \cdot \boldsymbol{v}_{1}=0 \\
& \boldsymbol{M}^{0}(1) \cdot \boldsymbol{v}_{1}=0 \\
& \boldsymbol{x}^{0}(1) \cdot\left(\boldsymbol{v}_{1} \times \boldsymbol{e}_{3}\right)=0 \\
& \boldsymbol{x}^{0}(1) \cdot \boldsymbol{v}_{1}= r^{0}(1)=\frac{1}{\kappa_{1}^{0}(1)}\left(1-\left(d_{33}^{0}(1)\right)^{2}\right), \\
& z^{0}(1)=1,
\end{aligned}
$$

where (65) is the $O(1)$ contribution from (31), with the radius of the helix also affected by the perturbation scheme, i.e., $r(s, t)=r^{0}(s)+\delta r^{t}(s, t)$.

\section{B. The $O(\delta)$ equations - linearisation}

The $O(\delta)$ part of the linear momentum equation (34) can be written as

$$
\begin{aligned}
\left(\boldsymbol{F}^{t}(s, t)\right)^{\prime} & +\boldsymbol{B}_{1}(s) \boldsymbol{F}^{t}(s, t)+\boldsymbol{B}_{2}(s) \boldsymbol{x}^{t}(s, t)+\boldsymbol{B}_{3}(s) \boldsymbol{\alpha}^{\prime}(s, t)+\boldsymbol{B}_{4}(s) \boldsymbol{\alpha}(s, t) \\
& =\boldsymbol{B}_{5}(s) \ddot{\boldsymbol{x}}^{t}(s, t)+\boldsymbol{B}_{6}(s) \dot{\boldsymbol{x}}^{t}(s, t),
\end{aligned}
$$

where the $3 \times 3$ matrices $\boldsymbol{B}_{i}(s)$ are given in Appendix A. Here we have expressed $\boldsymbol{F}^{t}$ relative to $\left\{\boldsymbol{d}_{1}^{0}, \boldsymbol{d}_{2}^{0}, \boldsymbol{d}_{3}^{0}\right\}$ and $\boldsymbol{x}^{t}$ relative to $\left\{\boldsymbol{e}_{1}, \boldsymbol{e}_{2}, \boldsymbol{e}_{3}\right\}$. For the $O(\delta)$ part of the angular momentum equation (35) we can write

$$
\begin{aligned}
\left(\boldsymbol{M}^{t}(s, t)\right)^{\prime} & +\boldsymbol{C}_{1}(s) \boldsymbol{M}^{t}(s, t)+\boldsymbol{C}_{2}(s) \boldsymbol{\alpha}^{\prime}(s, t)+\boldsymbol{C}_{3}(s) \boldsymbol{\alpha}(s, t)+\boldsymbol{C}_{4}(s) \boldsymbol{F}^{t}(s, t) \\
& =\boldsymbol{C}_{5}(s) \ddot{\boldsymbol{\alpha}}(s, t)+\boldsymbol{C}_{6}(s) \dot{\boldsymbol{\alpha}}(s, t),
\end{aligned}
$$


where the matrices $\boldsymbol{C}_{i}(s)$ are again given in Appendix A. $\boldsymbol{M}^{t}$ is expressed relative to $\left\{\boldsymbol{d}_{1}^{0}, \boldsymbol{d}_{2}^{0}, \boldsymbol{d}_{3}^{0}\right\}$. The 9 twist equations (37) at $O(\delta)$ are reduced to only 3 independent equations that relate $\boldsymbol{\kappa}^{t}$ and $\boldsymbol{\alpha}$ as

$$
\boldsymbol{\kappa}^{t}(s, t)=\boldsymbol{\alpha}^{\prime}(s, t)+\boldsymbol{\kappa}^{0}(s) \times \boldsymbol{\alpha}(s, t) .
$$

Introducing these relations into the $O(\delta)$ part of the constitutive relations gives

$$
\boldsymbol{M}^{t}(s, t)+\boldsymbol{D}_{1}(s) \boldsymbol{\alpha}^{\prime}(s, t)+\boldsymbol{D}_{2}(s) \boldsymbol{\alpha}(s, t)=\boldsymbol{D}_{3}(s) \dot{\boldsymbol{\alpha}}(s, t)+\boldsymbol{D}_{4}(s) \frac{\partial^{2}}{\partial s \partial t}(\boldsymbol{\alpha}(s, t)),
$$

where the matrices $\boldsymbol{D}_{i}(s)$ are given in Appendix A. Finally, the $O(\delta)$ part of equation (36) yields

$$
\left(\boldsymbol{x}^{t}(s, t)\right)^{\prime}=\alpha(s, t) \times \boldsymbol{d}_{3}^{0}(s) .
$$

Applying the perturbation scheme to the boundary conditions at $O(\delta)$, we obtain

$$
\begin{aligned}
\alpha_{1}(0, t) & =0, \\
\alpha_{3}(0, t) & =0, \\
x^{t}(0, t) & =0, \\
d_{13}^{0}(0) F_{1}^{t}(0, t)+d_{33}^{0}(0) F_{3}^{t}(0, t)+\left(d_{13}^{0}(0) F_{3}^{0}(0)-d_{33}^{0}(0) F_{1}^{0}(0)\right) \alpha_{2}(0, t) & =0, \\
F_{2}^{t}(0, t) & =0, \\
M_{2}^{t}(0, t) & =0, \\
x^{t}(1, t) & =0, \\
z^{t}(1, t) & =0, \\
\alpha_{1}(1, t) & =0, \\
\alpha_{3}(1, t) & =0, \\
M_{2}^{t}(1, t) & =0, \\
y^{t}(1, t)+\frac{2}{\kappa_{1}^{0}(1)}\left(\boldsymbol{d}_{3}^{0}(1) \cdot \boldsymbol{e}_{3}\right)\left(\boldsymbol{d}_{3}^{t}(1, t) \cdot \boldsymbol{e}_{3}\right)+\frac{1}{\kappa_{1}^{0}(1)^{2}}\left(1-\left(\boldsymbol{d}_{3}^{0}(1) \cdot \boldsymbol{e}_{3}\right)^{2}\right) \kappa_{1}^{t}(1, t) & =0 .
\end{aligned}
$$

After elimination of the $\kappa_{i}^{0}$ by means of (54), the set of 12 equations (67), (68), (170) and (71) together with the 12 boundary conditions (72) -(83)), with appropriate initial conditions form a well-posed initial-boundary-value problem. 


\section{STABILITY ANALYSIS}

Since we are interested in stability of solutions we look for solutions of the $O(\delta)$ equations of the form

$$
\begin{aligned}
\boldsymbol{x}^{t}(s, t) & =\hat{\boldsymbol{x}}^{t}(s) e^{\lambda t}, \\
\boldsymbol{\alpha}(s, t) & =\hat{\boldsymbol{\alpha}}(s) e^{\lambda t}, \\
\boldsymbol{F}^{t}(s, t) & =\hat{\boldsymbol{F}}^{t}(s) e^{\lambda t}, \\
\boldsymbol{M}^{t}(s, t) & =\hat{\boldsymbol{M}}^{t}(s) e^{\lambda t} .
\end{aligned}
$$

When these expressions are inserted into (67)-(71) a linear eigenvalue problem for a 12dimensional ODE is obtained in terms of the variables $\left(\hat{\boldsymbol{x}}^{t}, \hat{\boldsymbol{\alpha}}^{t}, \hat{\boldsymbol{F}}^{t}, \hat{\boldsymbol{M}}^{t}\right)$. The eigenvalue $\lambda$ measures the growth of small perturbations and is to be found as part of the solution. Eigenvalues come as complex conjugate pairs. A whirling state is unstable if at least one of the (in general infinitely many) $\lambda$ 's has positive real part.

To solve a real system of equations we split the eigenvalues and variables (eigenfunctions) into real and imaginary parts, $\lambda=\lambda_{r}+i \lambda_{i}, \hat{\boldsymbol{x}}^{t}=\hat{\boldsymbol{x}}_{r}^{t}+i \hat{\boldsymbol{x}}_{i}^{t}, \hat{\boldsymbol{\alpha}}^{t}=\hat{\boldsymbol{\alpha}}_{r}^{t}+i \hat{\boldsymbol{\alpha}}_{i}^{t}, \hat{\boldsymbol{F}}^{t}=\hat{\boldsymbol{F}}_{r}^{t}+i \hat{\boldsymbol{F}}_{i}^{t}$ and $\hat{\boldsymbol{M}}^{t}=\hat{\boldsymbol{M}}_{r}^{t}+i \hat{\boldsymbol{M}}_{i}^{t}$. The equations (67)-(71) along with the boundary conditions (72)(83), are similarly split into real and imaginary parts. Thus we end up with a doubled 24-dimensional linearised boundary-value problem.

\section{A. Stability of the straight rod - static magnetic buckling}

The trivial solution of the $O(1)$ equations (46)-(咕), representing a straight and untwisted rod, is given by

$$
\boldsymbol{x}(s)=s \boldsymbol{e}_{3}, \quad \boldsymbol{F}(s)=-T \boldsymbol{e}_{3}, \quad \boldsymbol{M}(s)=\mathbf{0}, \quad \boldsymbol{d}_{i}(s)=\boldsymbol{e}_{i} \quad(i=1,2,3), \quad s \in[0,1] .
$$

It satisfies the coat hanger boundary conditions. For the statics case $(\omega=0)$ without end force $(T=0)$ the $O(\delta)$ equations ([67), (68), (170), (71) about this trivial solution, on inserting (84) - (87), can be written as

$$
\begin{aligned}
& x^{\prime \prime \prime \prime}-\lambda^{2} P x^{\prime \prime}+\frac{\lambda^{2}}{R} x-\frac{B}{R} y^{\prime}=0, \\
& y^{\prime \prime \prime \prime}-\lambda^{2} P y^{\prime \prime}+\lambda^{2} y+B x^{\prime}=0, \\
& M_{3}^{\prime \prime}-\frac{2 \lambda^{2} P}{\Gamma} M_{3}=0,
\end{aligned}
$$


with boundary conditions

$$
x(0)=x(1)=x^{\prime \prime}(0)=x^{\prime \prime}(1)=y^{\prime \prime \prime}(0)=y(1)=y^{\prime}(0)=y^{\prime}(1)=0, \quad M_{3}^{\prime}(0)=0=M_{3}^{\prime}(1),
$$

while $F_{3} \equiv 0, z \equiv 0$. Note that the torsional $\left(M_{3}\right)$ modes decouple from the bending $(x, y)$ modes.

To find the static magnetic buckling loads we set $\lambda=0$. The bending equations then reduce to

$$
z^{\prime \prime \prime \prime \prime \prime \prime}+\frac{B^{2}}{R} z=0, \quad \text { for } \quad z=x^{\prime}
$$

subject to

$$
x(0)=x(1)=x^{\prime \prime}(0)=x^{\prime \prime}(1)=x^{\prime \prime \prime \prime}(0)=x^{\prime \prime \prime \prime}(1)=0 .
$$

On setting $z=e^{i k s}$ we obtain the characteristic equation $-k^{6}+B^{2} / R=0$ with solutions $k_{1,2}= \pm \beta, k_{3,4,5,6}= \pm \beta(1 \pm i \sqrt{3}) / 2$, where $\beta=B^{1 / 3} / R^{1 / 6}$. Application of the boundary conditions (92) to the general solution $z(s)=\sum_{j=1}^{6} a_{j} e^{i k_{j} s}$ leads to the remarkably simple condition:

$$
B=(n \pi)^{3} \sqrt{R}
$$

These critical loads correspond to pitchfork bifurcations where non-trivial solutions bifurcate from the trivial straight solution. We stress that the above calculation is only possible for the statics case. If $\omega \neq 0$ then the $x$ and $y$ equations do not decouple and no simple characteristic equation is obtained. However, bifurcating branches of helical solutions, and hence critical loads, can be computed explicitly, even for non-zero $\omega$; see Appendix B.

\section{B. Eigenvalues for the unperturbed problem $(T=0, \gamma=0, \omega=0, B=0)$}

We shall call the case where $T=0, \gamma=0, \omega=0$ and $B=0$ the unperturbed problem. For this problem explicit expressions can be obtained for the eigenvalues of the linearisation about the straight solution. The $x$ and $y$ equations in (89) decouple into two fourth-order beam equations:

$$
\begin{gathered}
x^{\prime \prime \prime \prime}-\lambda^{2} P x^{\prime \prime}+\frac{\lambda^{2}}{R} x=0, \\
y^{\prime \prime \prime \prime}-\lambda^{2} P y^{\prime \prime}+\lambda^{2} y=0,
\end{gathered}
$$


subject to boundary conditions (90). Since we anticipate imaginary eigenvalues we set $\lambda=i \mu, x=e^{i k s}, y=e^{i \kappa s}$, and find for the $x$ equation

$$
\begin{aligned}
& k_{1,2}= \pm\left(\frac{1}{2} \mu^{2} P+\frac{1}{2} \sqrt{\mu^{4} P^{2}+4 \mu^{2} / R}\right)^{1 / 2}=: \pm a, \\
& k_{3,4}= \pm i\left(\frac{1}{2} \sqrt{\mu^{4} P^{2}+4 \mu^{2} / R}-\frac{1}{2} \mu^{2} P\right)^{1 / 2}=: \pm i b,
\end{aligned}
$$

while for the $y$ equation

$$
\begin{aligned}
& \kappa_{1,2}= \pm\left(\frac{1}{2} \mu^{2} P+\frac{1}{2} \sqrt{\mu^{4} P^{2}+4 \mu^{2}}\right)^{1 / 2}=: \pm \alpha \\
& \kappa_{3,4}= \pm i\left(\frac{1}{2} \sqrt{\mu^{4} P^{2}+4 \mu^{2}}-\frac{1}{2} \mu^{2} P\right)^{1 / 2}=: \pm i \beta
\end{aligned}
$$

where $a, b, \alpha, \beta$ are non-negative real numbers. The general solutions are

$$
\begin{aligned}
& x(s)=A_{x} \sin a s+B_{x} \cos a s+C_{x} \sinh b s+D_{x} \cosh b s, \\
& y(s)=A_{y} \sin \alpha s+B_{y} \cos \alpha s+C_{y} \sinh \beta s+D_{y} \cosh \beta s .
\end{aligned}
$$

Application of the boundary conditions (90) leads to

$$
\sin a=0 \quad \text { and } \quad \beta \cos \alpha \sinh \beta+\alpha \sin \alpha \cosh \beta=0 .
$$

The first equation implies

$$
\mu= \pm \frac{n^{2} \pi^{2}}{\sqrt{n^{2} \pi^{2} P+1 / R}}, \quad n=1,2,3, \ldots
$$

The second equation is transcendental and needs to be solved numerically to obtain the eigenvalues $\mu$, for instance by using a Newton-Raphson scheme. Meanwhile, the torsional eigenvalues for the $M_{3}$ equation in (89) are given by

$$
\mu= \pm n \pi \sqrt{\frac{\Gamma}{2 P}}, \quad n=1,2,3, \ldots
$$

These are all the eigenvalues for the unperturbed problem. They will be used as starting values in the numerical procedure described next.

\section{Numerical procedure}

The main idea is to use the known eigenvalues in the unperturbed problem as starting values in a continuation procedure in order to compute the eigenvalues and corresponding 
eigenfunctions for general values of the parameters $T, \gamma, \omega$ and $B$. For this we use the welltested code AUTO [13] (specifically AUTO2000). AUTO solves boundary-value problems by means of orthogonal collocation. It requires a starting solution and can then trace out solution curves as a parameter of the problem is varied. Bifurcations are detected where branches of solutions intersect. At such points AUTO is able to switch branches and compute curves of bifurcating solutions.

Our procedure takes advantage of the fact that $\lambda$ appears only quadratically in the linearisation (67), (68), (70) and (171) if $\gamma=0$ and $\omega=0$. To explain the method consider the typical $O(\delta)$ equation

$$
z^{\prime \prime \prime \prime}-\lambda^{2} f(s) z^{\prime \prime}+\lambda^{2} g(s) z=0
$$

where $f$ and $g$ are functions of the $O(1)$ solution. Writing $z=x+i y, \lambda=\lambda_{r}+i \lambda_{i}$, we can decompose the $z$ equation into

$$
\begin{aligned}
& x^{\prime \prime \prime \prime}-\left(\lambda_{r}^{2}-\lambda_{i}^{2}\right) f(s) x^{\prime \prime}+2 \lambda_{r} \lambda_{i} f(s) y^{\prime \prime}+\left(\lambda_{r}^{2}-\lambda_{i}^{2}\right) g(s) x-2 \lambda_{r} \lambda_{i} g(s) y=0, \\
& y^{\prime \prime \prime \prime \prime}-\left(\lambda_{r}^{2}-\lambda_{i}^{2}\right) f(s) y^{\prime \prime}-2 \lambda_{r} \lambda_{i} f(s) x^{\prime \prime}+\left(\lambda_{r}^{2}-\lambda_{i}^{2}\right) g(s) y+2 \lambda_{r} \lambda_{i} g(s) x=0 .
\end{aligned}
$$

The important thing to note here is that these equations decouple into two identical equations if the eigenvalue is either imaginary $\left(\lambda_{r}=0\right)$ or real $\left(\lambda_{i}=0\right)$.

This suggests the following sequence of steps, involving boundary-value problem of increasing dimension, to compute eigenvalues of static or uniformly whirling solutions.

1. Consider the unperturbed problem of Section $\mathrm{VB}$ and, noting that all eigenvalues are purely imaginary, solve the 30-dimensional system of $18 O(1)$ equations and one 12dimensional system for the imaginary part of the $O(\delta)$ equations (cf. the $y$ equation in (96)). Set $\lambda_{r}=0$ and use $\lambda_{i}$ as the continuation parameter in AUTO in order to compute the eigenvalues (instead of solving the transcendental equations in Section VB). These eigenvalues will show up as branching points (BP), or pitchfork bifurcations, as eigenvalues by definition are those values for which non-zero BVP solutions exist. By symmetry it is only necessary to consider $\lambda_{i}>0$.

2. Keeping the same 30-dimensional system, switch branches at a BP to compute ('grow') the corresponding (imaginary) eigenfunction. Since the equations are linear the value of $\lambda_{i}$ will not change in this run. For later use we monitor the non-zero solution by means of some measure $\|.\|_{i}$ (not necesarily a proper norm) on the space $\left\{\hat{\boldsymbol{x}}_{i}^{t}, \hat{\boldsymbol{\alpha}}_{i}^{t}, \hat{\boldsymbol{F}}_{i}^{t}, \hat{\boldsymbol{M}}_{i}^{t}\right\}$ of imaginary linearised variables. 
3. Now consider the full system of 42 equations $(18 O(1)$ equations and two sets of $12-$ dimensional $O(\delta)$ equations (cf. (96) ). Fix the measure $\|.\|_{i}$ on the imaginary part and release $\lambda_{r}$ instead in order to compute the real eigenfunction (since the imaginary part of the solution is fixed there is only one branch of solutions through the starting point and there is nowhere else to go for the continuation but to 'grow' the real eigenfunction). Again we monitor this function by means of a suitable measure $\|.\|_{r}$. In this run neither $\lambda_{r}$ nor $\lambda_{i}$ will change.

This approach works because the solution obtained in step 2 also solves the full 42dimensional system when the extra 12 variables $\left(\hat{\boldsymbol{x}}_{r}^{t}, \hat{\boldsymbol{\alpha}}_{r}^{t}, \hat{\boldsymbol{F}}_{r}^{t}, \hat{\boldsymbol{M}}_{r}^{t}\right)$ are set to zero. This is a consequence of the fact that the real and imaginary parts of the $O(\delta)$ equations decouple if $\lambda_{r}=0$, as a result of the quadratic dependence of the eigenvalue problem on $\lambda(\mathrm{cf} .(96))$.

Steps 2 and 3 can be performed for as many of the BPs computed in step 1 as required and will give the corresponding eigenvalues and eigenfunctions. Once these have been obtained both measures $\|.\|_{r}$ and $\|.\|_{i}$ can be fixed and an extra system parameter such as $B$ or $\omega$ released in order to trace the eigenvalues (and hence monitor stability changes) as system parameters are varied. (Note that fixing $\|.\|_{r}$ and $\|.\|_{i}$ makes sense as eigenfunctions are only defined up to a multiplicative factor.)

The above 3-step procedure is not limited to linearisations about the trivial straight solution. It can be applied to any starting solution that has no eigenvalue with both $\lambda_{r}$ and $\lambda_{i}$ non-zero, as these would not be picked up in step 1. (It is of course no problem if eigenvalues become fully complex (Hopf bifurcation) in the course of further continuations.) For instance, we find that at the first critical $B$, given by (93), the lowest conjugate pair of eigenvalues $\pm \lambda_{i}$ goes to zero and becomes a real pair of eigenvalues, signalling a stability change of the straight rod. The (first-mode) solution bifurcating at this point is stable with all eigenvalues being imaginary and the above procedure can be applied to find the eigenvalues.

We end this section with a few comments:

(i) There are infinitely many eigenvalues and the above procedure only finds the lowest order ones. This is of course a limitation of any numerical scheme. We find that 


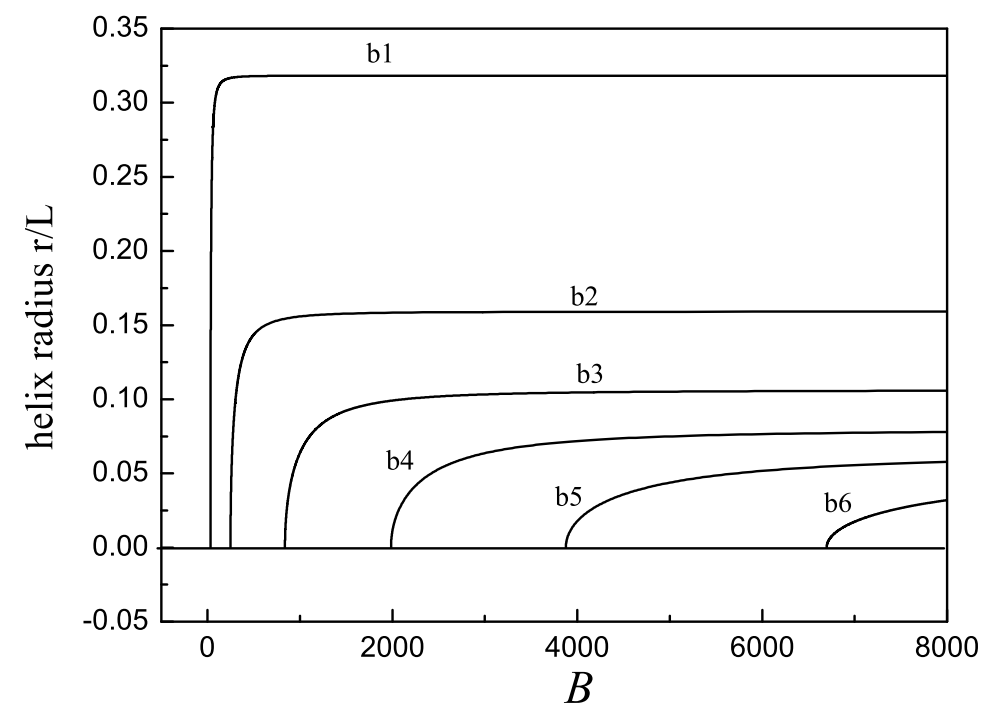

FIG. 4: Bifurcation diagram for a stationary isotropic rod subject to coat hanger boundary conditions $(\omega=0)$.

eigenvalues vary slowly with system parameters, suggesting that stability is governed by the lowest-order eigenvalues. We typically consider 5 or 6 eigenvalues.

(ii) Note that in steps 1 and 2 above we could not have taken the full 42-dimensional system of equations as that would have made the branching points (pitchfork bifurcations) degenerate and AUTO would not detect a BP. This is because if $\lambda_{r}=0$ (or $\left.\lambda_{i}=0\right)$ the two sets of 12-dimensional linearised equations are identical (cf. (96)).

\section{NUMERICAL RESULTS}

\section{A. The statics case $(\omega=0)$}

Fig. 4 shows the bifurcation diagram obtained by varying the magnetic field parameter $B$ in the isotropic case $(R=1)$. Throughout this entire section the dimensionless parameters taken, unless stated otherwise, are those listed in Table I, where realistic dimensional parameters are also given. Pitchfork bifurcations on the trivial branch occur at $B P 1=31.01$, $B P 2=248.05, B P 3=837.17$, etc., in agreement with (93) (only the positive- $r$ branch is shown). The bifurcating solutions are found to be exact helices and therefore the dimensionless helical radius $r / L$ is used as solution measure on the vertical axis. A slight complication 
TABLE I: Parameters used for the coat hanger boundary conditions.

\begin{tabular}{|c|c||c|c|}
\hline$L$ & $5 \mathrm{~m}$ & $P$ & 0.001 \\
\hline$A$ & $3 \times 10^{-5} \mathrm{~m}^{2}$ & $R$ & 1 \\
\hline$E$ & $30 \times 10^{9} \mathrm{~N} / \mathrm{m}^{2}$ & $\Gamma$ & 0.76923 \\
\hline$I_{1}=I_{2}$ & $9 \times 10^{-11} \mathrm{~m}^{4}$ & & \\
\hline
\end{tabular}
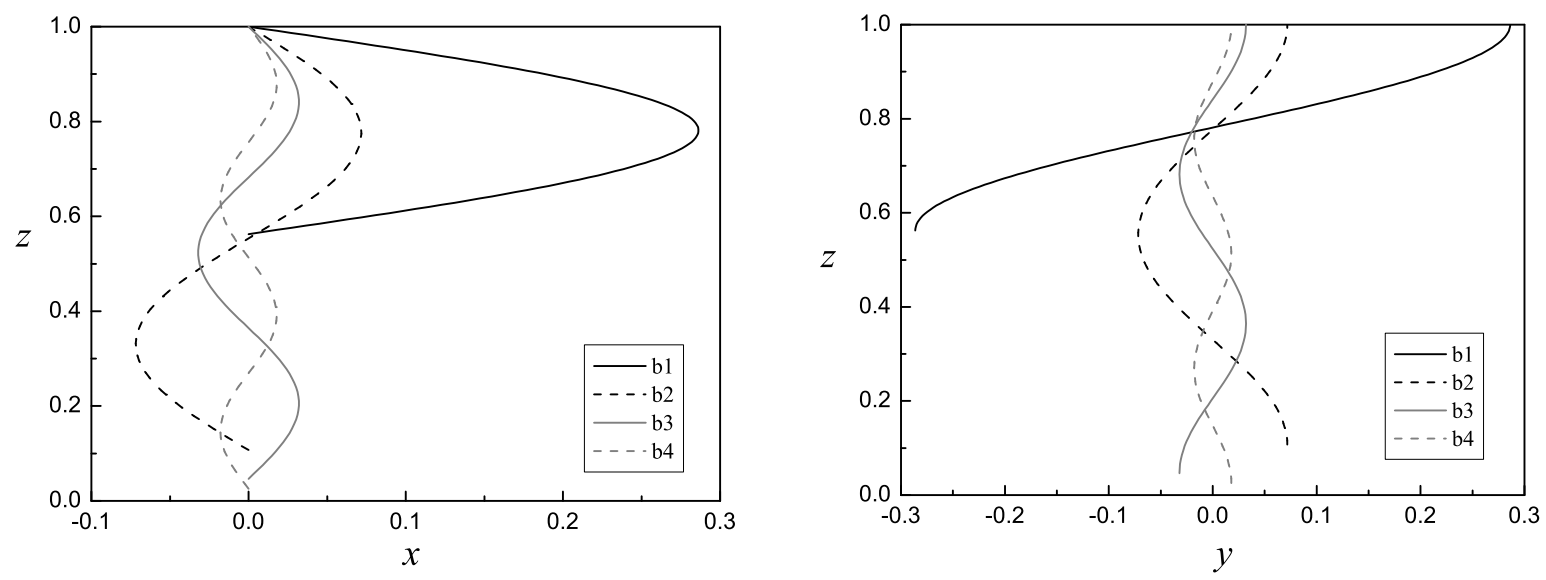

FIG. 5: The $\left\{\boldsymbol{e}_{1}-\boldsymbol{e}_{3}\right\}$ and $\left\{\boldsymbol{e}_{2}-\boldsymbol{e}_{3}\right\}$ projections of the first 4 helical modes at constant curvature $\kappa=8$. Values of $B$ are: 61.68 (b1), 270.69 (b2), 868.72 (b3) and 2024.70 (b4).

in computing this diagram occurs because of the denominator in (32), which is zero for the straight rod. However, this problem is easily resolved by replacing boundary condition (31) by $\boldsymbol{x}(L) \cdot \boldsymbol{v}_{1}=0$ along the trivial branch and switching back to (31) once an incipient non-trivial solution has been obtained.

Fig. [5 shows $\left\{\boldsymbol{e}_{1}-\boldsymbol{e}_{3}\right\}$ and $\left\{\boldsymbol{e}_{2}-\boldsymbol{e}_{3}\right\}$ projections of bifurcating solutions along the first four branches, taken at constant curvature $\kappa=8$. It was noted in Section IIB that the coat hanger boundary conditions with $\chi=0$ (i.e., parallel end supports $\boldsymbol{v}_{0}$ and $\boldsymbol{v}_{1}$ ) only allow helices of a half-integer number, $n$, of helical turns. We find that each successive bifurcating solution in Fig. 4 has one more half helical turn. For large $B$ the solutions approach a circular shape in the $z=1$ plane, with corresponding value $r / L=1 /(n \pi)$ along the vertical axis in Fig. 4. The bifurcating branches have handedness. That is, regardless of the sign of $r$, the bifurcating solutions are right-handed helices if, as here, $B>0$ and would be 


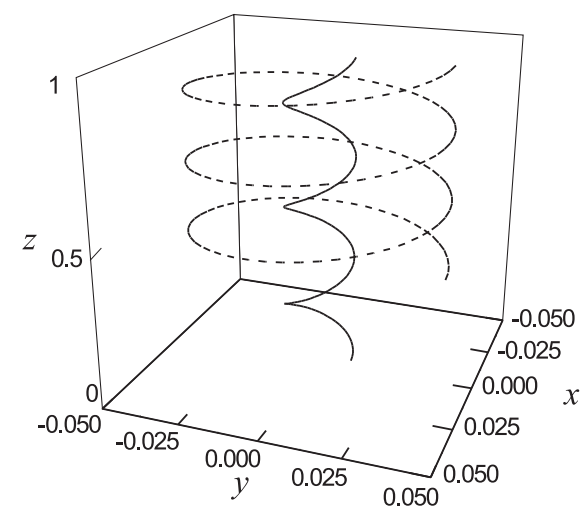

FIG. 6: 3D view of two helices on branch b6.

(a)

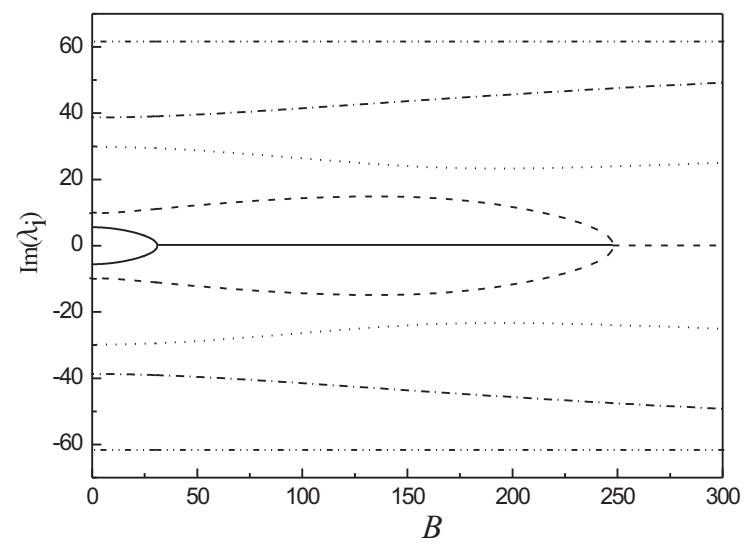

(b)



FIG. 7: Evolution of imaginary (a) and real (b) parts of the first five pairs of eigenvalues along the trivial solution, from $B=0$ to $B>B P 2$.

left-handed helices if $B<0$, i.e., had we run $B$ in the other direction. Fig. 66 shows threedimensional views of two solutions along the sixth branch. They have three full turns and nicely illustrate the exact helical shape.

Fig. 7 show the evolution of the imaginary and real parts, respectively, of the first five pairs of eigenvalues along the trivial solution, from $B=0$ to $B>B P 2$. At successive pitchfork bifurcations, pairs of imaginary eigenvalues collide at zero and become real, one of the eigenvalues of the pair with positive real part, signalling that the trivial solution becomes unstable at $B=B P 1$, while a further loss of stability occurs at $B P 2$ where a second pair of eigenvalues becomes real.

Fig. 8 shows the evolution of the first five pairs of imaginary eigenvalues when switching 


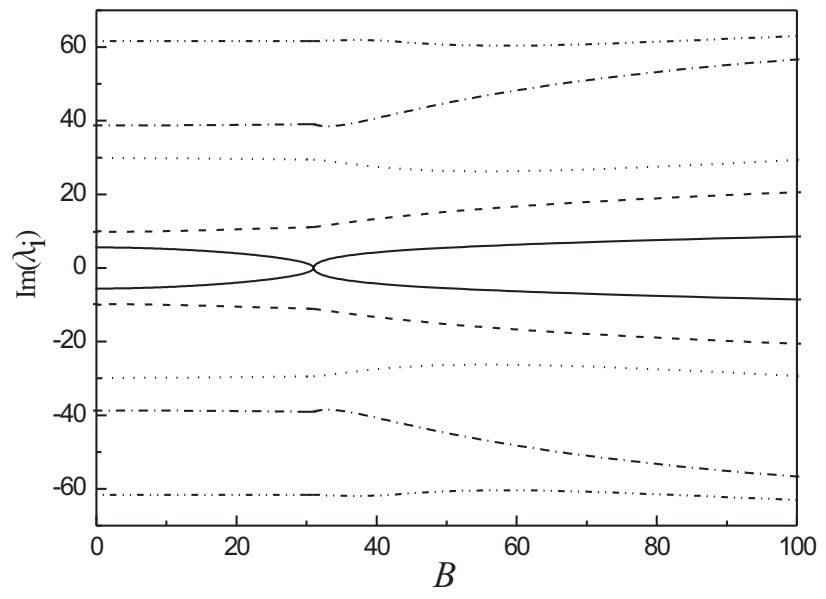

FIG. 8: Imaginary part evolution of the first five pairs of eigenvalues along the trivial solution, from $B=0$ to $B=B P 1$ and then switching to branch b1. All real parts of the eigenvalues are zero.

(a)

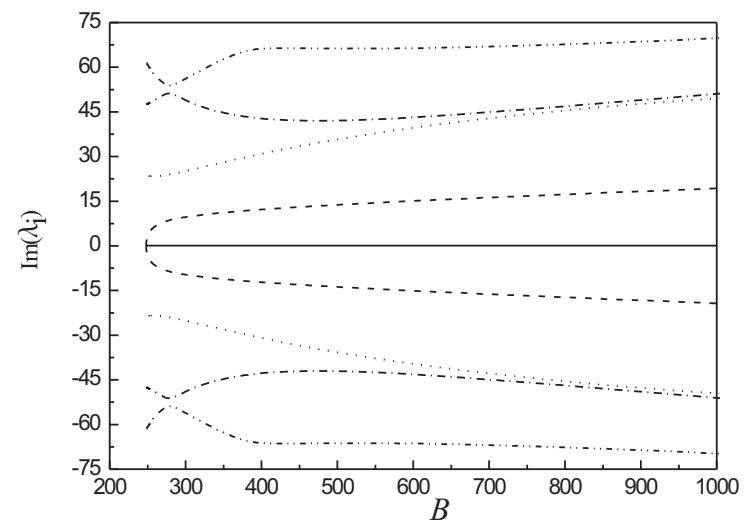

(b)

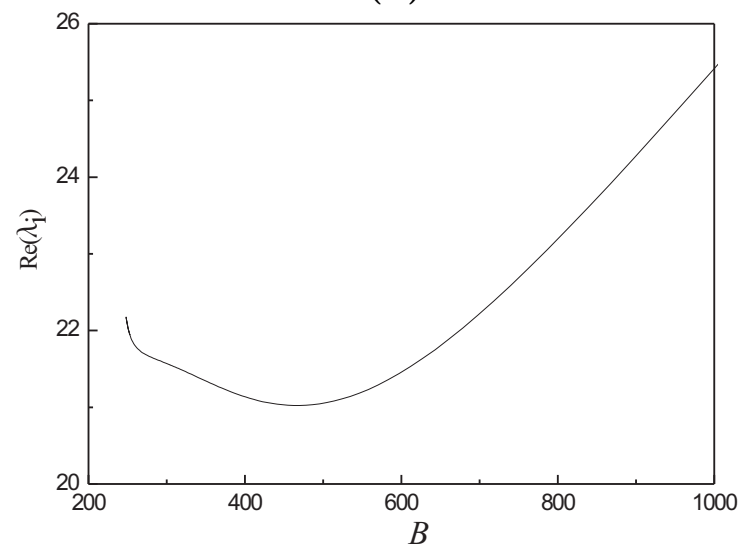

FIG. 9: Evolution of the imaginary (a) and (positive) real (b) parts of the first five pairs of eigenvalues along branch b2.

at BP1 from the trivial branch to b1, which is found to be stable. As expected, solutions along b2 are found to be unstable (see Figs $9 a, b$ ).

\section{B. Whirling solutions $(\omega \neq 0)$ - Hopf bifurcations}

Next we consider whirling motions in which the supports $\boldsymbol{v}_{0}$ and $\boldsymbol{v}_{1}$ are spun about $\boldsymbol{k}$ with constant angular velocity $\omega$. Solutions remain helical, as expected. Fig. 10 shows the 


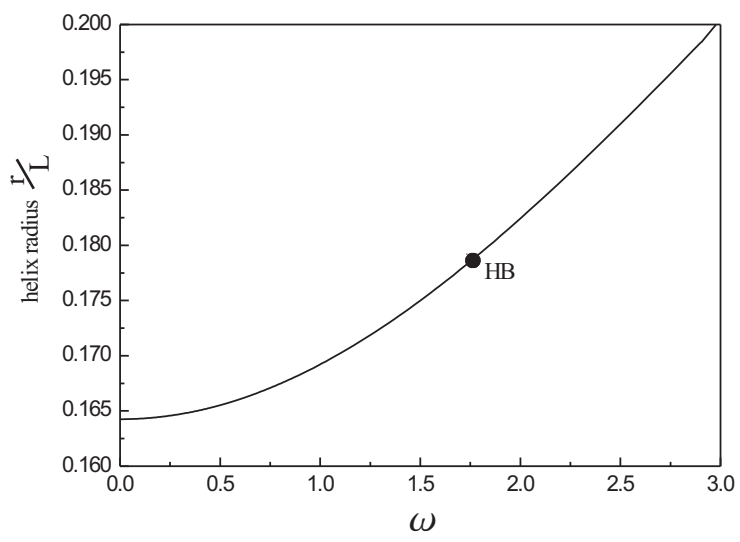

FIG. 10: Effect of $\omega$ on the helical radius of the solution along b1 in Fig. 4 at $B=35$. The dot indicates a Hopf bifurcation where the real parts of two complex conjugate eigenvalues become positive (see Fig. 14(a)) and the solution loses stability.

effect of $\omega$ on a solution taken on the first bifurcating branch (b1) of Fig. 4. The helical radius increases with $\omega$. Fig. 11 shows the bifurcation diagram for a fixed value of $\omega=2$. Pitchfork bifurcations along the trivial branch occur at $B=29.75,247.44,836.80$, etc.

The angular velocity tends to destabilise the helical solutions (see Fig. 12, where the eigenvalues are shown as a function of $\omega$ ). We now introduce damping by continuing in the

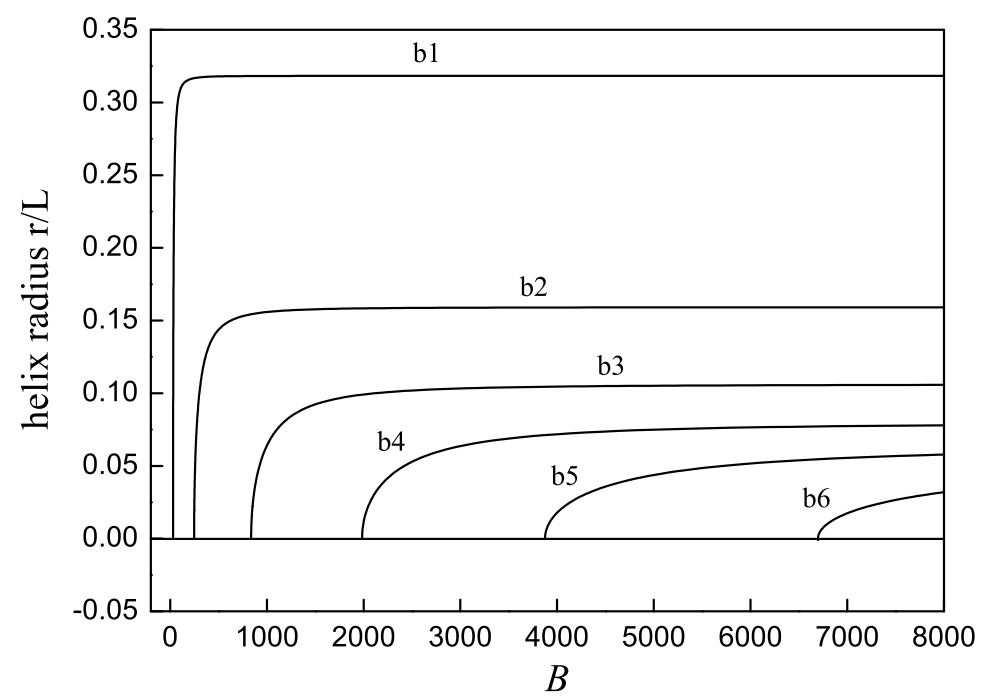

FIG. 11: Bifurcation diagram for a whirling isotropic rod subject to coat hanger boundary conditions $(\omega=2)$. 
(a)

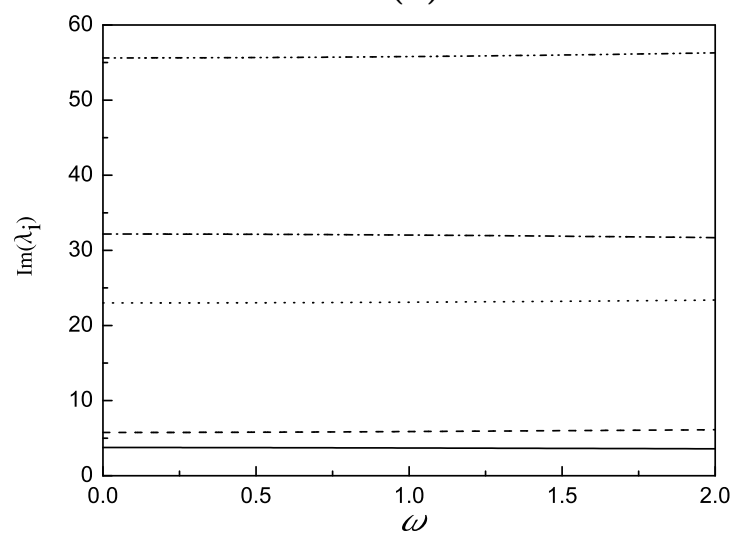

(c)



(e)

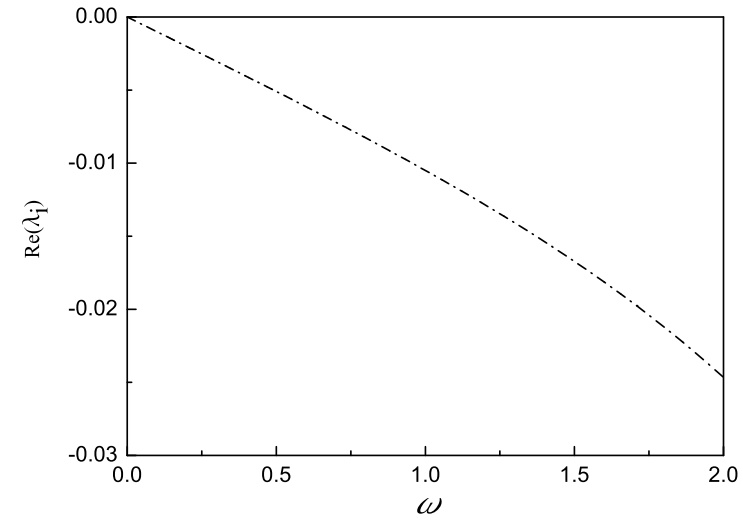

(b)

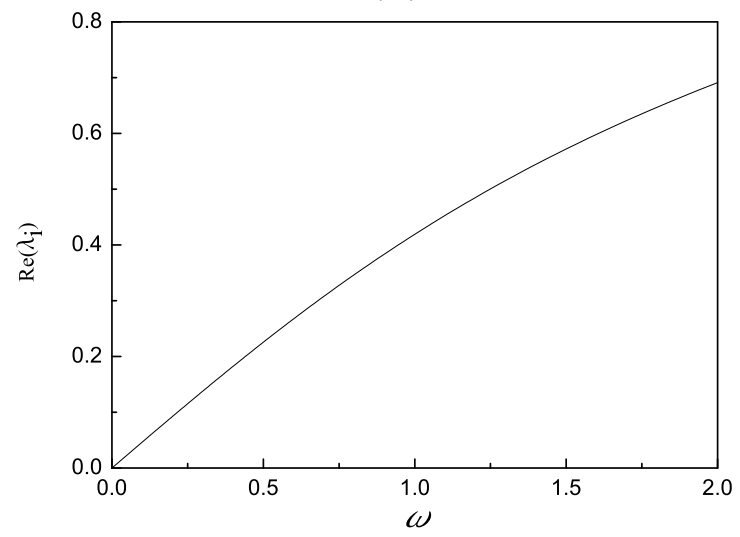

(d)

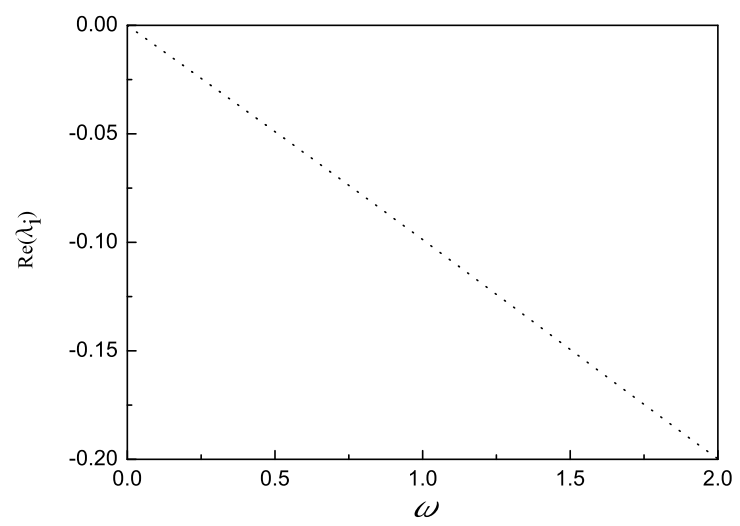

(f)

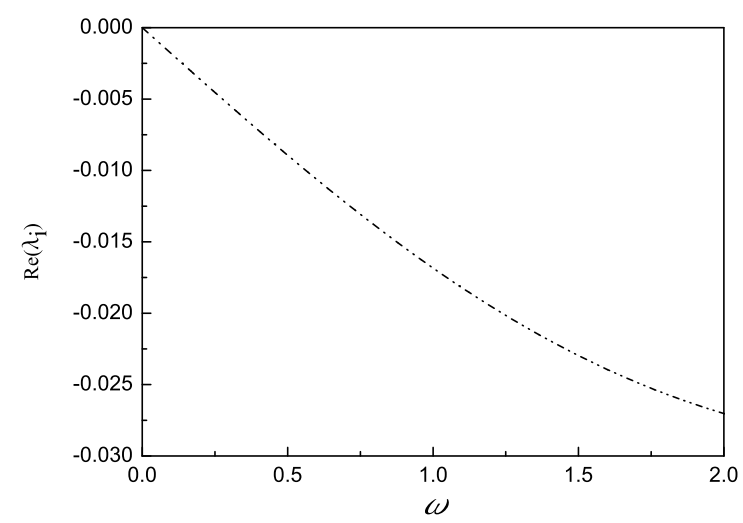

FIG. 12: Evolution of imaginary (a) and real part (b-f) of the first five pairs of eigenvalues with respect to $\omega$ for a solution of the first branch b1 of Fig. 4 for $B=35$, bifurcation diagram in Fig. 10. Note that first panel only represents the positive imaginary eigenvalue, as the negative is symmetric with respect to the real axis. 
(a)

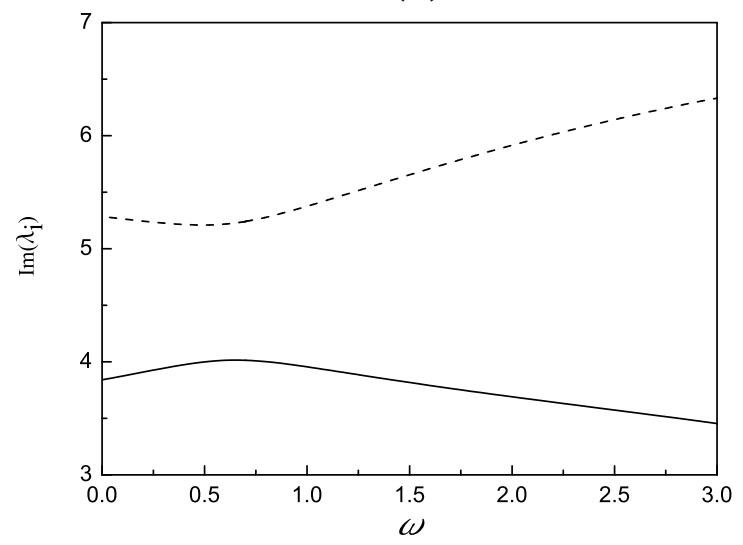

(b)

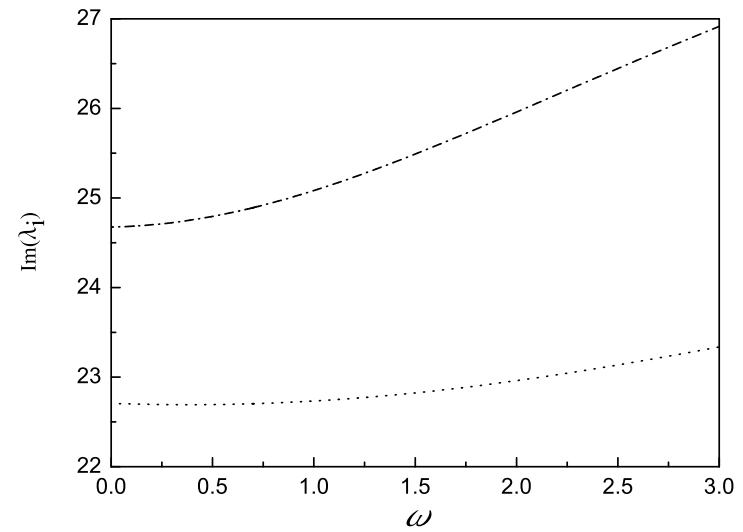

(c)

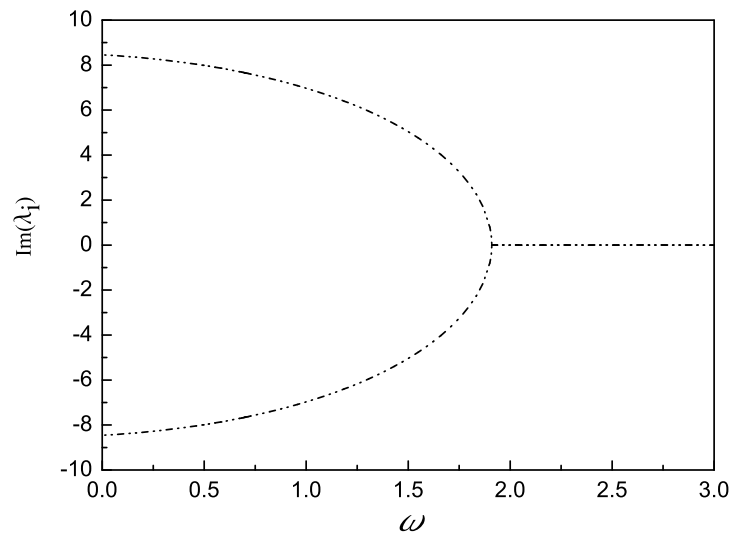

FIG. 13: Evolution with respect to $\omega$ of the imaginary part of the first five pairs of eigenvalues for a solution along b1 at $B=35$ and $\gamma=0.05$. The first two panels only show the positive imaginary part.

parameter $\gamma$, and to further investigate the stability of the first mode we fix $\gamma=0.05$ [10] and perform continuation in $\omega$. Figs 13 and 14 show that at $\omega=0$ the solution is stable and that around $\omega=1.75$ the real part of the first eigenvalue becomes positive. Thus the system loses stability in a Hopf bifurcation. The point where this occurs is indicated in the bifurcation diagram in Fig. 10, which is still valid as $\gamma$ has no effect on relative equilibria. Fig. 15 shows curves of Hopf bifurcations in the $\omega$ - $B$ parameter plane for various values of $\gamma$. At the end points of these curves (indicated by dots) the Hopf bifurcation coalesces with the pitchfork bifurcation in which the curve b1 is created. Consequently, the whirling helical solution is stable below these curves and unstable above. For small $\gamma$ the curve collapses 
(a)



(b)



(c)

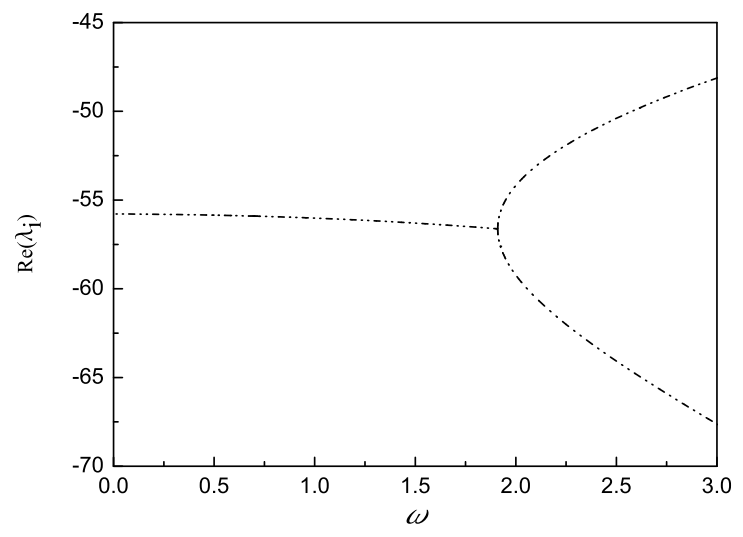

FIG. 14: Evolution with respect to $\omega$ of the real part of the first five pairs of eigenvalues for a solution along b1 at $B=35$ and $\gamma=0.05$.

onto the line $\omega=0$. Note that for relatively large $\omega$ damping has a stabilising effect, but that for small positive and for negative $\omega$ damping has a destabilising effect. The latter behaviour is known from the classical linear stability theory of gyroscopic systems [14].

\section{The stationary anisotropic rod - Hamiltonian-Hopf bifurcations}

It is known that in the absence of damping and inertial effects (i.e., $\gamma=0, \omega=0$ ) the equations for a rod in a magnetic field have a Hamiltonian structure [15]. In Hamiltonian systems a common mechanism for loss of stability is through a so-called Hamiltonian-Hopf bifurcation [16]. In this bifurcation two imaginary eigenvalues move on the imaginary axis, meet at some non-zero value and then leave the axis to become a real pair, as illustrated 


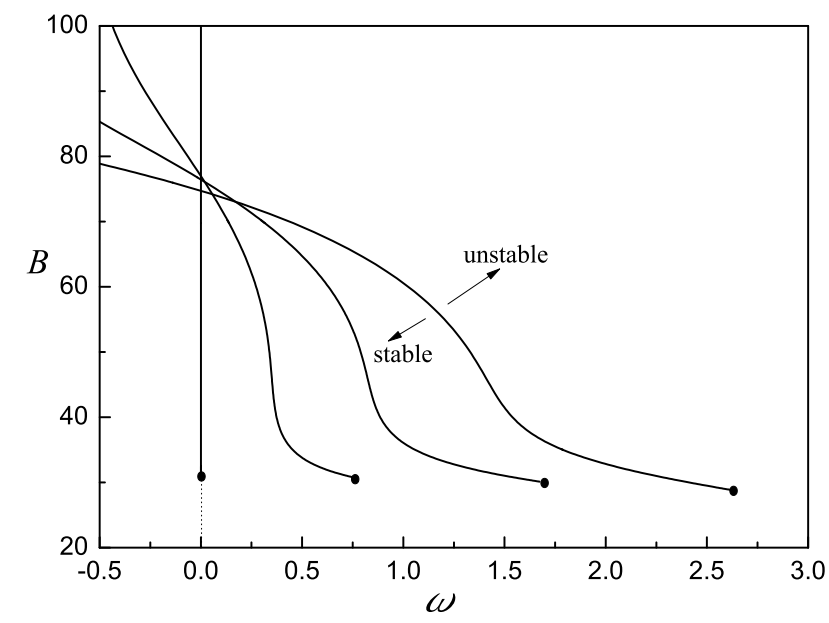

FIG. 15: Loci of Hopf bifurcations along b1 for different values of the damping parameter $\gamma$ : from left to right, $\gamma=0, \gamma=0.01, \gamma=0.025$ and $\gamma=0.05$. Dots indicate points where the Hopf bifurcation coalesces with the primary pitchfork bifurcation.

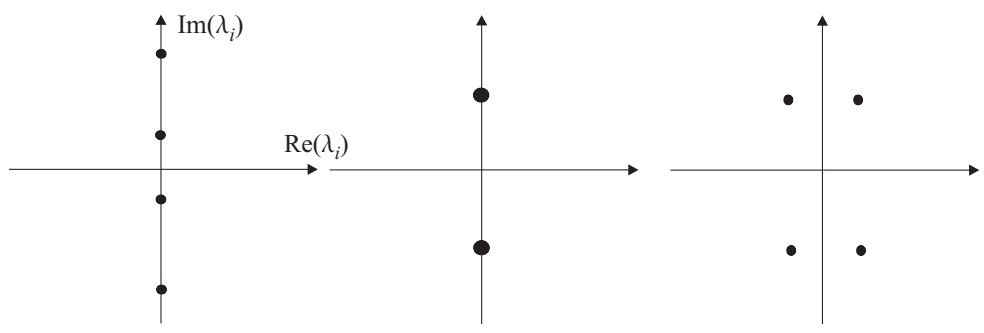

FIG. 16: Eigenvalue behaviour in a Hamiltonian-Hopf bifurcation.

in Fig. 16, Since eigenvalues come as conjugate pairs this event involves four eigenvalues. In structural problems Hamiltonian-Hopf bifurcations usually mark oscillatory instabilities such as flutter.

No Hamiltonian-Hopf bifurcation was found in our study of the isotropic rod in Section VIA. However, after breaking the cross-sectional symmetry by taking $R<1$ HamiltonianHopf bifurcations are found to occur under $B$ continuation. Fig. 17 shows a bifurcation diagram for $R=0.5512$. Pitchfork bifurcations along the trivial branch are found at $B=$ 23.058, 184.56, 622.55, etc., in agreement with (93). Bifurcating solutions are not pure helices. Figs 18 and 19 show the eigenvalue behaviour for a solution on the first bifurcating branch, b1, in Fig. 17. The first and second eigenvalues collide at $B=23.26$, where the solution becomes unstable. The fifth and sixth eigenvalues then collide at $B=23.39$ but 




FIG. 17: Bifurcation diagram for a stationary anisotropic rod subject to coat hanger boundary conditions $(\omega=0, R=0.5512)$. Bifurcating solutions are not exact helices.

shortly after become imaginary again in a reverse Hamiltonian-Hopf bifurcation at $B=$ 23.76. The first and second eigenvalues then also become imaginary again at $B=54.79$, restabilising the solution (as far as we checked no other eigenvalues cause instability, see Fig. 19, where $\lambda_{4}, \lambda_{5}$ and $\lambda_{7}$ are shown).

Fig. 20 shows loci of Hamiltonian-Hopf bifurcations in the $R-B$ parameter plane. At the end points of the curves the Hamiltonian-Hopf bifurcation coalesces with the pitchfork bifurcation. The curve of pitchfork bifurcations is given by (93) and is included in dotted lines. We have instability inside the region bounded by solid and dotted curves. Note that the stability of the b1 solution is almost entirely, but not completely, determined by the first two eigenvalues.

\section{CONCLUSION}

We have shown that whirling current-carrying transversely isotropic rods bifurcate under increasing magnetic field (or current) into exact helical shapes provided one applies what we call coat hanger boundary conditions. The first bifurcating branch, containing solutions with half a helical turn, is stable while higher-order branches are all unstable.

We stress that the stability analysis of Section 5.1 together with the helical analysis in 
(a)



(c)

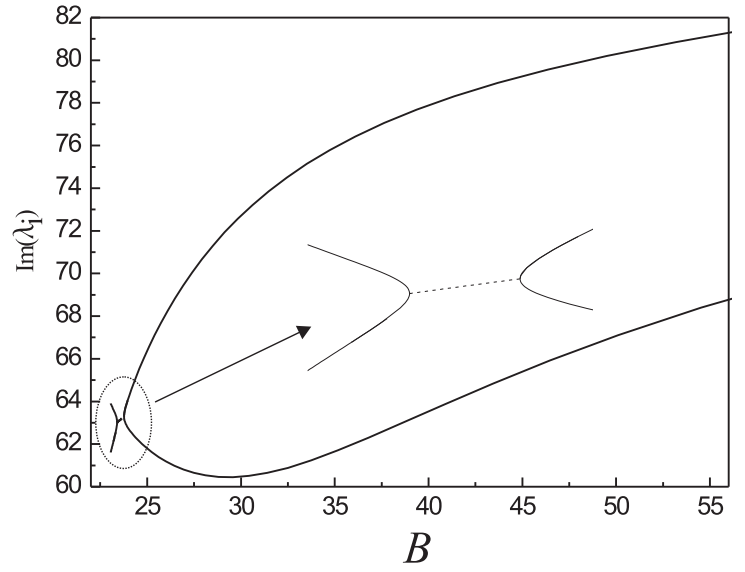

(b)

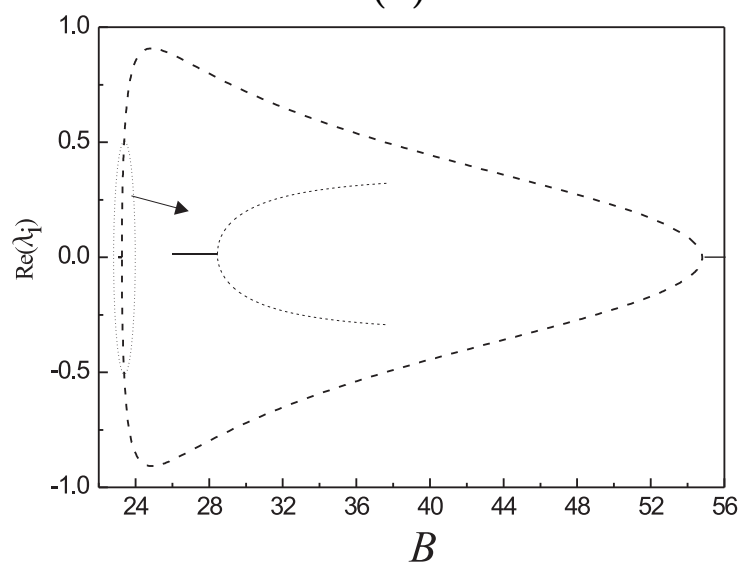

(d)



FIG. 18: Evolution with respect to $B$ of the first and second ((a) and (b)) and fifth and sixth ((c) and $(\mathrm{d})$ ) eigenvalues for an initially stable anisotropic-rod solution on the first bifurcating branch (b1). Both pairs of eigenvalues undergo a Hamiltonian-Hopf followed by a reverse HamiltonianHopf bifurcation. $(\gamma=0, \omega=0, R=0.55$.

Appendix B gives a complete picture of magnetically-induced helical buckling subject to these boundary conditions. In Section 5.1 we show that the critical loads for a straight rod are given by equation (93). These bifurcation points are non-degenerate and valid for both isotropic and anisotropic rods. The analysis says nothing, however, about the type of solutions that bifurcate. They could be helices or not. In Appendix B we then compute branches of helical solutions and show that, for isotropic rods (i.e., $R=1$ ), they intersect the trivial branch of straight rod solutions precisely at the critical loads computed in Section 5.1. Taken together these results prove that for an isotropic rod all solutions bifurcating 
(a)

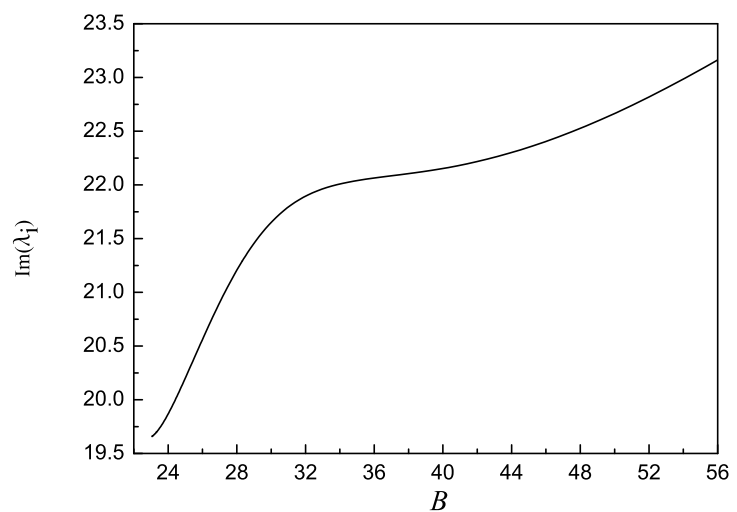

(b)

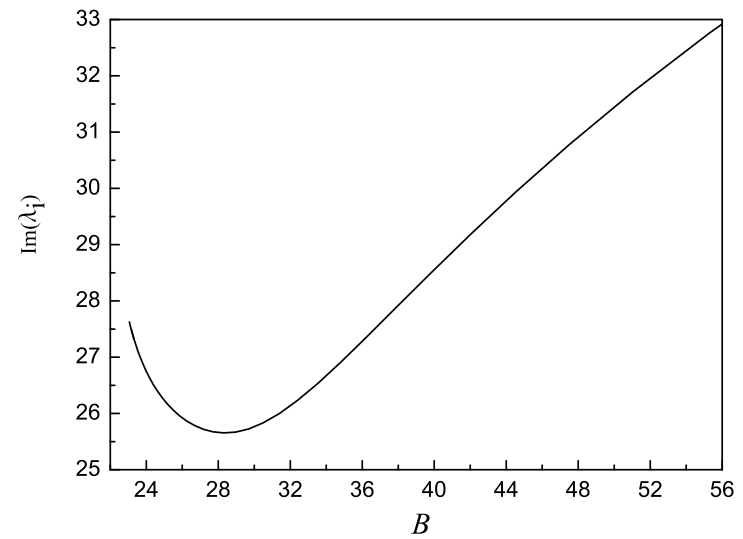

(c)

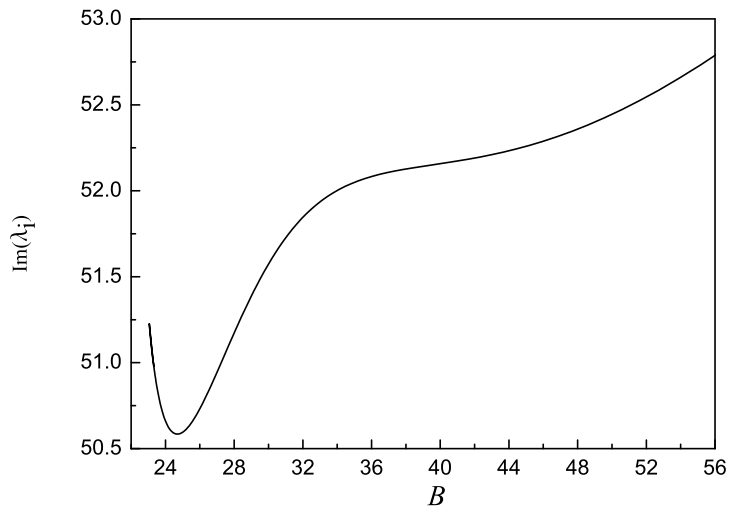

FIG. 19: Evolution with respect to $B(\gamma=0$ and $\omega=0)$ of the imaginary part (real part equals zero) of the third, fourth and seventh pairs of eigenvalues for non-circular cross-section, $R=0.55$.

from the straight rod are helical. For an anisotropic rod, on the other hand, the critical loads are still given by (93) (with $R \neq 1$ ) but no branch of helical solutions in Appendix B is found to intersect the trivial branch and therefore the bifurcating solutions are non-helical, as confirmed numerically in Section 6.3.

We have also investigated the stability of post-buckling solutions and found Hopf bifurcations where stable helical solutions lose stability. In the case of a non-rotating anisotropic rod we found secondary instabilities given by Hamiltonian-Hopf bifurcations. Unfortunately, our method allows us only to study stationary or quasi-stationary (whirling) solutions, so it is not clear what type of stable solutions occur after these secondary bifurcations. To investigate this one would have to do simulations based on direct discretisation of the PDEs (14) and (15). 


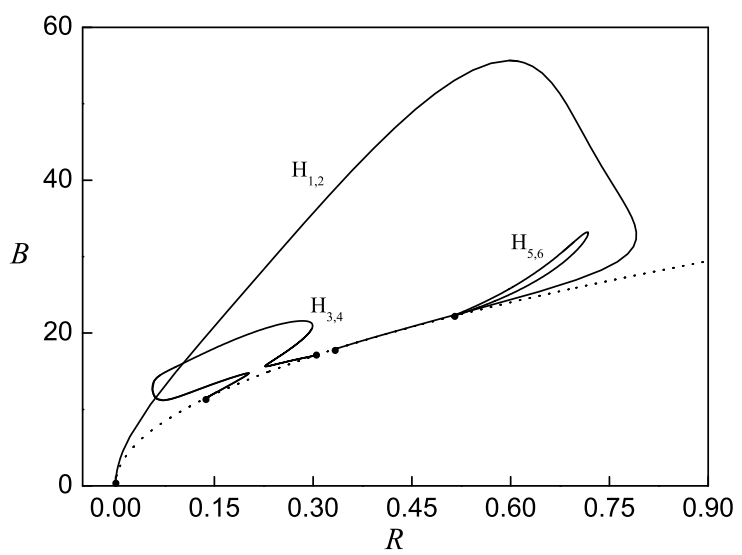

FIG. 20: Hamiltonian-Hopf loci for b1 solution at $\omega=0, R=0.55$. All curves terminate on the dotted curve of pitchfork bifurcations given by (93). We have instability of the solution inside the regions bounded by solid and dotted lines. $H_{i j}$ labels the curve generated by the $i$ th and $j$ th eigenvalue.

For whirling isotropic rods subject to welded boundary conditions magnetic buckling is described by a doubly-degenerate pitchfork bifurcation (see [6], where we also showed that the same is true for non-rotating states of anisotropic rods). This is because the equations are invariant under rotation about the axis ( $\boldsymbol{e}_{3}$ axis) of the supports. This symmetry property complicated Wolfe's analysis (for non-rotating states of isotropic rods), which had to take account of the variational nature of the problem to prove existence of non-trivial bifurcating states [5]. By contrast, the coat hanger boundary conditions here introduced break the $S^{1}$ symmetry down to $Z_{2}$ symmetry (reflection symmetry along $\boldsymbol{v}_{0}$ and $\boldsymbol{v}_{1}$ ), and no problems in the application of standard results from bifurcation theory should arise. Indeed, we find the (isolated) critical values of the magnetic field to be given by a remarkably simple explicit expression.

Helical solutions are often used and studied in applications of elastic rods or filaments. These solutions are incompatible with the common (aligned) clamped, pinned, Cardan joint and other boundary conditions. Boundary conditions are therefore often ignored in these studies, making it impossible to do a stability analysis. Here we have introduced a set of boundary conditions that does allow for helical solutions, not only in magnetic buckling but also in traditional buckling due to compression, twist or whirl. These boundary conditions allow one to study exact helical solutions in finite-length rods. We have also proposed a 
mechanical device that can be used for generating helical solutions in the laboratory.

\section{Acknowledgement}

J.V. wishes to thank the Andalusian Regional Ministry for financial support through the Excellence Research Programme, under the FQM-4239 project.

[1] H.H. Woodson, J.R. Melcher, Electromechanical Dynamics, Part II: Fields, Forces, and Motion, John Wiley \& Sons, New York, 1968.

[2] P. Wolfe, Equilibrium states of an elastic conductor in a magnetic field: A paradigm of bifurcation theory, Trans. Am. Math. Soc. 278 (1983) 377-387.

[3] P. Wolfe, Rotating states of an elastic conductor, in: Physical Mathematics and Nonlinear Partial Differential Equations, J. Lightbourne, S. Rankin (Eds.), Dekker, New York, 1985, pp. 213-222.

[4] T.J. Healey, Large rotating states of a conducting elastic wire in a magnetic field: subtle symmetry and multiparameter bifurcation, J. Elasticity 24 (1990) 211-227.

[5] P. Wolfe, Bifurcation theory of an elastic conducting rod in a magnetic field, Quart. J. Mech. Appl. Math. 41(2) (1988) 265-279.

[6] J. Valverde, G.H.M. van der Heijden, Magnetically-induced buckling of a whirling conducting rod with applications to electrodynamic space tethers, Journal of Nonlinear Science 20 (2010), 309-339.

[7] A. Goriely, M. Tabor, Nonlinear dynamics of filaments I. Dynamical instabilities, Physica D 105 (1997) 20-44.

[8] S.S. Antman, Nonlinear Problems of Elasticity, Springer-Verlag, Berlin, 1995.

[9] B.D. Coleman, E.H. Dill, M. Lembo, Z. Lu, I. Tobias, On the dynamics of rods in the theory of Kirchhoff and Clebsch, Arch. Rat. Mech. Anal. 121 (1993) 339-359.

[10] J. Valverde, J.L. Escalona, J. Domínguez, A.R. Champneys, Stability and bifurcation analysis of a spinning space tether, Journal of Nonlinear Science 16(5) (2006) 507-542.

[11] J.D. Jackson, Classical Electrodynamics, 2nd edition, John Wiley \& Sons, New York, 1975. 
[12] W.B. Fraser, D.M. Stump, Yarn twist in the ring-spinning balloon, Proc. R. Soc. Lond. A 454 (1998) 707-723.

[13] E.J. Doedel, A.R. Champneys, T.R. Fairgrieve, Yu.A. Kuznetsov, B. Sandstede, X.J. Wang, AUTO2000: Continuation and bifurcation software for ordinary differential equations (available by anonymous ftp from ftp.cs.concordia.ca/pub/doedel/auto), 2000.

[14] H. Ziegler, Principles of Structural Stability, Blaisdell, Waltham MA, 1968.

[15] D. Sinden, G.H.M. van der Heijden, Integrability of a conducting elastic rod in a magnetic field, J. Phys. A: Math. Theor. 41 (2008) 045207 (16pp).

[16] J.C. van der Meer, The Hamiltonian-Hopf bifurcation, Springer-Verlag, Berlin, 1985. 


\section{Appendix A: Matrices for the linearisation}

The matrices $\boldsymbol{B}_{i}$ appearing in equation (67) are given by

$$
\begin{aligned}
& \boldsymbol{B}_{1}=\left(\begin{array}{ccc}
0 & -\kappa_{3}^{0} & \kappa_{2}^{0} \\
\kappa_{3}^{0} & 0 & -\kappa_{1}^{0} \\
-\kappa_{2}^{0} & \kappa_{1}^{0} & 0
\end{array}\right) \\
& \boldsymbol{B}_{2}=\omega^{2}\left(\begin{array}{lll}
d_{11}^{0} & d_{12}^{0} & 0 \\
d_{21}^{0} & d_{22}^{0} & 0 \\
d_{31}^{0} & d_{32}^{0} & 0
\end{array}\right) \\
& \boldsymbol{B}_{3}=\left(\begin{array}{ccc}
0 & F_{3}^{0} & -F_{2}^{0} \\
-F_{3}^{0} & 0 & F_{1}^{0} \\
F_{2}^{0} & -F_{1}^{0} & 0
\end{array}\right) \\
& \boldsymbol{B}_{4}=\left(\begin{array}{ccc}
F_{2}^{0} \kappa_{2}^{0}+F_{3}^{0} \kappa_{3}^{0}-B\left(d_{22}^{0} d_{11}^{0}-d_{21}^{0} d_{12}^{0}\right) & \left(F_{3}^{0}\right)^{\prime}-F_{1}^{0} \kappa_{2}^{0} & -\left(F_{2}^{0}\right)^{\prime}-F_{1}^{0} \kappa_{3}^{0} \\
-\left(F_{3}^{0}\right)^{\prime}-F_{2}^{0} \kappa_{1}^{0} & F_{3} \kappa_{3}^{0}+F_{1}^{0} \kappa_{1}^{0}-B\left(d_{22}^{0} d_{11}^{0}-d_{21}^{0} d_{12}^{0}\right) & \left(F_{1}^{0}\right)^{\prime}-F_{2}^{0} \kappa_{3}^{0} \\
\left(F_{2}^{0}\right)^{\prime}-F_{3} \kappa_{1}^{0}-B\left(d_{22}^{0} d_{31}^{0}-d_{21}^{0} d_{32}^{0}\right) & -\left(F_{1}^{0}\right)^{\prime}-F_{3}^{0} \kappa_{2}^{0}+B\left(d_{12}^{0} d_{31}^{0}-d_{11}^{0} d_{32}^{0}\right) & F_{1}^{0} \kappa_{1}^{0}+F_{2}^{0} \kappa_{2}^{0}
\end{array}\right), \\
& \boldsymbol{B}_{5}=\left(\begin{array}{lll}
d_{11}^{0} & d_{12}^{0} & d_{13}^{0} \\
d_{21}^{0} & d_{22}^{0} & d_{23}^{0} \\
d_{31}^{0} & d_{32}^{0} & d_{33}^{0}
\end{array}\right), \\
& \boldsymbol{B}_{6}=2 \omega\left(\begin{array}{lll}
d_{12}^{0} & -d_{11}^{0} & 0 \\
d_{22}^{0} & -d_{21}^{0} & 0 \\
d_{32}^{0} & -d_{31}^{0} & 0
\end{array}\right) \text {. }
\end{aligned}
$$

Matrices $\boldsymbol{C}_{i}$ appearing in equation (68) are given by

$$
\begin{gathered}
\boldsymbol{C}_{1}=\boldsymbol{B}_{1}, \\
\boldsymbol{C}_{2}=\left(\begin{array}{ccc}
0 & M_{3}^{0} & -M_{2}^{0} \\
-M_{3}^{0} & 0 & M_{1}^{0} \\
M_{2}^{0} & -M_{1}^{0} & 0
\end{array}\right),
\end{gathered}
$$




$$
\boldsymbol{C}_{3}=\left(\begin{array}{ccc}
C_{3}^{11} & C_{3}^{12} & C_{3}^{13} \\
C_{3}^{21} & C_{3}^{22} & C_{3}^{23} \\
C_{3}^{31} & C_{3}^{32} & C_{3}^{33}
\end{array}\right)
$$

where

$$
\begin{aligned}
& C_{3}^{11}=M_{3}^{0} \kappa_{3}^{0}+M_{2}^{0} \kappa_{2}^{0}-P\left(\boldsymbol{\omega} \cdot \boldsymbol{d}_{3}^{0}\right)\left(\boldsymbol{d}_{2}^{0} \times \boldsymbol{\omega} \cdot \boldsymbol{d}_{1}^{0}\right)-P\left(\boldsymbol{\omega} \cdot \boldsymbol{d}_{2}^{0}\right)\left(\boldsymbol{d}_{3}^{0} \times \boldsymbol{\omega} \cdot \boldsymbol{d}_{1}^{0}\right), \\
& C_{3}^{12}=\left(M_{3}^{0}\right)^{\prime}-M_{1}^{0} \kappa_{2}^{0}-P R\left(\boldsymbol{\omega} \cdot \boldsymbol{d}_{1}^{0}\right)\left(\boldsymbol{d}_{1}^{0} \times \boldsymbol{\omega} \cdot \boldsymbol{d}_{3}^{0}\right), \\
& C_{3}^{13}=-\left(M_{2}^{0}\right)^{\prime}-M_{1}^{0} \kappa_{3}^{0}-F_{1}^{0}+P R\left(\boldsymbol{\omega} \cdot \boldsymbol{d}_{1}^{0}\right)\left(\boldsymbol{d}_{1}^{0} \times \boldsymbol{\omega} \cdot \boldsymbol{d}_{2}^{0}\right)+P\left(\boldsymbol{\omega} \cdot \boldsymbol{d}_{1}^{0}\right)\left(\boldsymbol{d}_{2}^{0} \times \boldsymbol{\omega} \cdot \boldsymbol{d}_{1}^{0}\right), \\
& C_{3}^{21}=-\left(M_{3}^{0}\right)^{\prime}-M_{2}^{0} \kappa_{1}^{0}+P\left(\boldsymbol{\omega} \cdot \boldsymbol{d}_{2}^{0}\right)\left(\boldsymbol{d}_{2}^{0} \times \boldsymbol{\omega} \cdot \boldsymbol{d}_{3}^{0}\right), \\
& C_{3}^{22}=M_{3}^{0} \kappa_{3}^{0}+M_{1}^{0} \kappa_{1}^{0}+P R\left(\boldsymbol{\omega} \cdot \boldsymbol{d}_{3}^{0}\right)\left(\boldsymbol{d}_{1}^{0} \times \boldsymbol{\omega} \cdot \boldsymbol{d}_{2}^{0}\right)+P R\left(\boldsymbol{\omega} \cdot \boldsymbol{d}_{1}^{0}\right)\left(\boldsymbol{d}_{3}^{0} \times \boldsymbol{\omega} \cdot \boldsymbol{d}_{2}^{0}\right), \\
& C_{3}^{23}=\left(M_{1}^{0}\right)^{\prime}-M_{2}^{0} \kappa_{3}^{0}-F_{2}^{0}-P R\left(\boldsymbol{\omega} \cdot \boldsymbol{d}_{2}^{0}\right)\left(\boldsymbol{d}_{1}^{0} \times \boldsymbol{\omega} \cdot \boldsymbol{d}_{2}^{0}\right)-P\left(\boldsymbol{\omega} \cdot \boldsymbol{d}_{2}^{0}\right)\left(\boldsymbol{d}_{2}^{0} \times \boldsymbol{\omega} \cdot \boldsymbol{d}_{1}^{0}\right), \\
& C_{3}^{31}=\left(M_{2}^{0}\right)^{\prime}-M_{3}^{0} \kappa_{1}^{0}+F_{1}^{0}-P\left(\boldsymbol{\omega} \cdot \boldsymbol{d}_{3}^{0}\right)\left(\boldsymbol{d}_{2}^{0} \times \boldsymbol{\omega} \cdot \boldsymbol{d}_{3}^{0}\right), \\
& C_{3}^{32}=-\left(M_{1}^{0}\right)^{\prime}-M_{3}^{0} \kappa_{2}^{0}+F_{2}^{0}+P R\left(\boldsymbol{\omega} \cdot \boldsymbol{d}_{3}^{0}\right)\left(\boldsymbol{d}_{1}^{0} \times \boldsymbol{\omega} \cdot \boldsymbol{d}_{3}^{0}\right), \\
& C_{3}^{33}=M_{2}^{0} \kappa_{2}^{0}+M_{1}^{0} \kappa_{1}^{0}+P(1-R)\left(\boldsymbol{\omega} \cdot \boldsymbol{d}_{2}^{0}\right)\left(\boldsymbol{d}_{1}^{0} \times \boldsymbol{\omega} \cdot \boldsymbol{d}_{3}^{0}\right)+P(1-R)\left(\boldsymbol{\omega} \cdot \boldsymbol{d}_{1}^{0}\right)\left(\boldsymbol{d}_{2}^{0} \times \boldsymbol{\omega} \cdot \boldsymbol{d}_{3}^{0}\right),
\end{aligned}
$$

$$
\begin{gathered}
\boldsymbol{C}_{4}=\left(\begin{array}{ccc}
0 & -1 & 0 \\
1 & 0 & 0 \\
0 & 0 & 0
\end{array}\right), \\
\boldsymbol{C}_{5}=P\left(\begin{array}{lll}
1 & 0 & 0 \\
0 & R & 0 \\
0 & 0 & (1+R)
\end{array}\right),
\end{gathered}
$$

$\boldsymbol{C}_{6}=2 P\left(\begin{array}{ccc}\left(\boldsymbol{d}_{2}^{0} \times \omega \times \boldsymbol{d}_{3}^{0}\right) \cdot \boldsymbol{d}_{1}^{0} & 0 & -\left(\boldsymbol{d}_{2}^{0} \times \omega \times \boldsymbol{d}_{1}^{0}\right) \cdot \boldsymbol{d}_{1}^{0} \\ 0 & -R\left(\boldsymbol{d}_{1}^{0} \times \omega \times \boldsymbol{d}_{3}^{0}\right) \cdot \boldsymbol{d}_{2}^{0} & R\left(\boldsymbol{d}_{1}^{0} \times \omega \times \boldsymbol{d}_{2}^{0}\right) \cdot \boldsymbol{d}_{2}^{0} \\ \left(\boldsymbol{d}_{2}^{0} \times \omega \times \boldsymbol{d}_{3}^{0}\right) \cdot \boldsymbol{d}_{3}^{0}-R\left(\boldsymbol{d}_{1}^{0} \times \omega \times \boldsymbol{d}_{3}^{0}\right) \cdot \boldsymbol{d}_{3}^{0} & R\left(\boldsymbol{d}_{1}^{0} \times \omega \times \boldsymbol{d}_{2}^{0}\right) \cdot \boldsymbol{d}_{3}^{0}-\left(\boldsymbol{d}_{2}^{0} \times \omega \times \boldsymbol{d}_{1}^{0}\right) \cdot \boldsymbol{d}_{3}^{0}\end{array}\right)$

Matrices $\boldsymbol{D}_{i}$ appearing in equation (70) are given by 


$$
\begin{aligned}
& \boldsymbol{D}_{1}=\left(\begin{array}{ccc}
-1 & 0 & 0 \\
0 & -R & 0 \\
0 & 0 & -\frac{\Gamma(1+R)}{2}
\end{array}\right) \\
& \boldsymbol{D}_{2}=\left(\begin{array}{ccc}
0 & \kappa_{3}^{0} & -M_{2}^{0}-(1-R) \kappa_{2}^{0} \\
-\kappa_{3}^{0} & 0 & M_{1}^{0}-(1-R) \kappa_{1}^{0} \\
M_{2}^{0}-\kappa_{2}^{0}\left(R-\frac{\Gamma(1+R)}{2}\right) & -M_{1}^{0}+\kappa_{1}^{0}\left(1-\frac{\Gamma(1+R)}{2}\right) & 0
\end{array}\right) \\
& \boldsymbol{D}_{3}=\gamma\left(\begin{array}{ccc}
0 & -\kappa_{3}^{0} & \kappa_{2}^{0} \\
R \kappa_{3}^{0} & 0 & -R \kappa_{1}^{0} \\
-\frac{\Gamma(1+R)}{2} \kappa_{2}^{0} & \frac{\Gamma(1+R)}{2} \kappa_{1}^{0} & 0
\end{array}\right) \\
& \boldsymbol{D}_{4}=-\gamma \boldsymbol{D}_{1} \text {. }
\end{aligned}
$$

All the $\kappa_{i}^{0}$ in the above can be expressed in terms of the moments $M_{i}^{0}$ by means of the constitutive relations (54). 


\section{Appendix B: Helical solutions}

If we assume a helical shape for the magnetically buckled rod then we can derive exact solutions as well as buckling loads. For this it is convenient to introduce Euler angles $(\theta, \psi$, $\phi)$ and relate the director frame $\left\{\boldsymbol{d}_{1}, \boldsymbol{d}_{2}, \boldsymbol{d}_{3}\right\}$ to the rotating frame $\left\{\boldsymbol{e}_{1}, \boldsymbol{e}_{2}, \boldsymbol{e}_{3}\right\}$ as follows:

$$
\begin{aligned}
\boldsymbol{d}_{1}= & (\sin \phi \cos \psi+\cos \phi \cos \theta \sin \psi) \boldsymbol{e}_{1}+(\sin \phi \sin \psi-\cos \phi \cos \theta \cos \psi) \boldsymbol{e}_{2} \\
& +\cos \phi \sin \theta \boldsymbol{e}_{3}, \\
\boldsymbol{d}_{2}= & (\cos \phi \cos \psi-\sin \phi \cos \theta \sin \psi) \boldsymbol{e}_{1}+(\cos \phi \sin \psi+\sin \phi \cos \theta \cos \psi) \boldsymbol{e}_{2} \\
& -\sin \phi \sin \theta \boldsymbol{e}_{3}, \\
\boldsymbol{d}_{3}= & -\sin \theta \sin \psi \boldsymbol{e}_{1}+\sin \theta \cos \psi \boldsymbol{e}_{2}+\cos \theta \boldsymbol{e}_{3} .
\end{aligned}
$$

The rate of change of the director frame is given by

$$
\boldsymbol{d}_{i}^{\prime}=\boldsymbol{\kappa} \times \boldsymbol{d}_{i} \quad(i=1,2,3),
$$

where $\boldsymbol{\kappa}=\kappa_{1} \boldsymbol{d}_{1}+\kappa_{2} \boldsymbol{d}_{2}+\kappa_{3} \boldsymbol{d}_{3}$ is the curvature vector. Inverting (98) and using orthogonality of the directors gives

$$
\kappa_{1}=\boldsymbol{d}_{2}{ }^{\prime} \cdot \boldsymbol{d}_{3}, \quad \kappa_{2}=\boldsymbol{d}_{3}{ }^{\prime} \cdot \boldsymbol{d}_{1}, \quad \kappa_{3}=\boldsymbol{d}_{1}{ }^{\prime} \cdot \boldsymbol{d}_{2},
$$

which, on inserting (97), yields

$$
\begin{aligned}
& \kappa_{1}=\psi^{\prime} \sin \theta \cos \phi-\theta^{\prime} \sin \phi, \\
& \kappa_{2}=-\psi^{\prime} \sin \theta \sin \phi-\theta^{\prime} \cos \phi \\
& \kappa_{3}=\phi^{\prime}+\psi^{\prime} \cos \theta .
\end{aligned}
$$

So for the total curvature $\kappa$ we find

$$
\kappa^{2}=\kappa_{1}^{2}+\kappa_{2}^{2}=\theta^{\prime 2}+\psi^{\prime 2} \sin ^{2} \theta .
$$

Now we make the assumption of a helical centreline and uniformly rotating directors by taking $\theta$ to be a constant and setting $\psi^{\prime}=\Omega, \phi^{\prime}=\nu$ ( $\Omega$ and $\nu$ constants). (That $\phi^{\prime}$ may be taken constant is not a priori clear. However, performing the following calculation without this assumption, while still taking $\psi^{\prime}$ constant, one is quickly led to conclude that $\phi^{\prime}$ is in 
fact constant, so to simplify the presentation we assume constancy of $\phi^{\prime}$ from the start.) By integrating $\boldsymbol{x}^{\prime}=\boldsymbol{d}_{3}$ we then find for the shape

$$
\begin{aligned}
& x(s)=r \cos (\Omega s+\psi(0)), \\
& y(s)=r \sin (\Omega s+\psi(0)), \quad \text { where } \quad r=\frac{\sin \theta}{\Omega}=\frac{\sin ^{2} \theta}{\kappa}, \\
& z(s)=L+(s-L) \cos \theta,
\end{aligned}
$$

and with this solution the boundary conditions (18) - (29) reduce to the four kinematical conditions

$$
\cos (\Omega L+\psi(0))=0, \quad \cos (\chi+\psi(0))=0, \quad \sin (\nu L+\phi(0))=0, \quad \sin \phi(0)=0,
$$

where we have allowed for a non-zero angle $\chi$ between the axes $\boldsymbol{v}_{0}$ and $\boldsymbol{v}_{1}$ (see Fig. 3). Compatibility of the director and fixed frame for the case of a straight (but twisted) rod (i.e., with $\theta=0)$ requires

$$
\psi(0)+\phi(0)=\frac{\pi}{2}-\chi, \quad \psi(L)+\phi(L)=\frac{\pi}{2}
$$

(both frames are aligned at $s=L$ and have a relative rotation $\chi$ about $\boldsymbol{e}_{3}=\boldsymbol{k}$ at $s=0$ ). Equations (103) and (104) imply

$$
\Omega=\frac{n \pi+\chi}{L}, \quad \nu=-\frac{n \pi}{L}, \quad \phi(0)=0, \pi, \quad \psi(0)=\frac{\pi}{2}-\chi-\phi(0) \quad(n \in \mathbb{Z}) .
$$

To find the reduced equilibrium equation we integrate the force balance equation (16) to get (letting $F_{x}=\boldsymbol{F} \cdot \boldsymbol{e}_{1}, F_{y}=\boldsymbol{F} \cdot \boldsymbol{e}_{2}, F_{z}=\boldsymbol{F} \cdot \boldsymbol{e}_{3}$ )

$$
\begin{aligned}
& F_{x}(s)=-I B_{0} r \sin (\Omega s+\psi(0))-\frac{r \rho \omega^{2} A}{\Omega} \sin (\Omega s+\psi(0)), \\
& F_{y}(s)=I B_{0} r \cos (\Omega s+\psi(0))+\frac{r \rho \omega^{2} A}{\Omega} \cos (\Omega s+\psi(0)), \\
& F_{z}(s)=T .
\end{aligned}
$$

and insert these expressions, together with (100) and the constitutive relations (10) with $\gamma_{v}=0$, in the moment balance equation (17) written out in the director frame. The twisting moment is directly given by its constitutive relation to be

$$
M_{3}=G J \kappa_{3}=G J\left(\phi^{\prime}+\psi^{\prime} \cos \theta\right)=G J(\nu+\Omega \cos \theta),
$$


i.e., a constant. In the isotropic case $\left(I_{1}=I_{2}=: I_{0}\right)$ the moment balance equation for $M_{3}$ is therefore identically satisfied, while the equations for $M_{1}$ and $M_{2}$ each give

$$
\left(E I_{0}-G J\right) \Omega \cos \theta-G J \nu-\frac{I B_{0}}{\Omega^{2}} \cos \theta+\frac{T}{\Omega}-\frac{\rho \omega^{2} A}{\Omega^{3}} \cos \theta+\frac{\rho \omega^{2} I}{\Omega} \cos \theta=0,
$$

with $\Omega$ and $\nu$ given by (105). The torque $M$ of the lower axis $\boldsymbol{v}_{0}$ about $\boldsymbol{k}$ is given by

$$
M=M_{z}(0)=E I_{0} \Omega \sin ^{2} \theta+G J(\nu+\Omega \cos \theta) \cos \theta .
$$

Thus we have an infinite set of post-buckling helical solution branches parametrised by

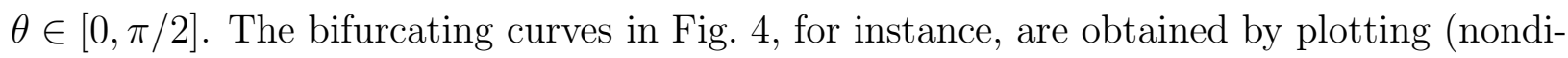
mensionalised) $r$ against $I B_{0}$ for $n=1, \ldots, 6$, using equations (102) and (107). The handedness of helical solutions is determined by the sign of $\Omega$, right-handed for $\Omega>0$. The two solutions for $\phi(0)$ in (105) correspond to the two branches emanating from the pitchfork bifurcations, both with the same handedness but with opposite signs for both $\kappa_{1}$ and $\kappa_{2}$.

The buckling condition is obtained by setting $\theta=0$ :

$$
I B_{0}=\frac{E I_{0}}{L^{3}}(n \pi+\chi)^{3}-\frac{G J \chi}{L^{3}}(n \pi+\chi)^{2}+\frac{T}{L}(n \pi+\chi)-\frac{\rho \omega^{2} A L}{n \pi+\chi}+\frac{\rho \omega^{2} I}{L}(n \pi+\chi),
$$

or, for $\chi=0$ and in dimensionless parameters,

$$
B=n^{3} \pi^{3}+n \pi \bar{T}-\frac{\bar{\omega}^{2}}{n \pi}+n \pi \bar{\omega}^{2} P .
$$

This expression shows that an applied tension $(\bar{T})$ and inertia $(P)$ stiffen the rod against helical buckling, while whirl $(\bar{\omega})$ softens it, as expected. The critical values of $B$ agree with the pitchfork bifurcations in Figs 4 and 11, and with (93) for the present case of an isotropic rod. Formula (109) is of course also valid in the absence of a magnetic field $\left(B_{0}=0\right)$, in which case it gives critical values for buckling due to compression $(T)$, twist $(\chi)$ or whirl $(\omega)$.

In the anisotropic case $\left(I_{1} \neq I_{2}\right)$ the moment balance equation for $M_{3}$ becomes $0=$ $\left(E \Omega^{2}+\rho \omega^{2}\right)\left(I_{2}-I_{1}\right) \sin ^{2} \theta \sin \phi \cos \phi$, whose only solution compatible with the boundary conditions (105) is $\phi \equiv 0$ or $\pi$. But this implies $\nu=0$ and hence, by (105), $n=0$ and $\Omega=\chi / L$. We conclude that in the case $\chi=0$, i.e., in the case of parallel axes $\boldsymbol{v}_{0}$ and $\boldsymbol{v}_{1}$, an anisotropic rod cannot buckle into a helical solution. This agrees with our numerical results, which show that the solutions bifurcating at the critical loads (93) when $R \neq 1$ are non-helical. 